\title{
Mapping mesoscale heterogeneity in the plastic deformation of a copper single crystal
}

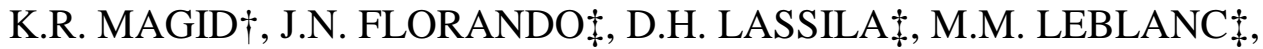
N. TAMURA $\S$, AND J.W. MORRIS, JR. $\dagger^{*}$

$\dagger$ Dept. Materials Science and Engineering, Univ. California, Berkeley, CA 94720

\$ Lawrence Livermore National Laboratory, Livermore, CA 94551

$\S$ Advanced Light Source, Lawrence Berkeley National Laboratory, Berkeley, CA 94720

*Corresponding author. Email: jwmorris@berkeley.edu

\begin{abstract}
The work reported here is part of a 'multiscale characterization' study of heterogeneous deformation patterns in metals. A copper single crystal was oriented for single slip in the (111)[ 101] slip system and tested to $\sim 10 \%$ strain in roughly uniaxial compression. The macroscopic strain field was monitored during the test by optical 'image correlation'. The strain field was measured on orthogonal surfaces, one of which (the $\mathrm{x}$-face) was oriented perpendicular to [ $1 \overline{2} 1]$ and contained the [ $\overline{101}$ ] direction of the preferred slip system. The macroscopic strain developed in an inhomogeneous pattern of broad, crossed shear bands in the $\mathrm{x}$-face. One, the primary band, lay parallel to (111). The second, the 'conjugate' band, was oriented perpendicular to (111) with an overall ( 101) habit that contains no common slip plane of the fcc crystal. The mesoscopic deformation pattern was explored with selected area diffraction, using a focused synchrotron radiation polychromatic beam with a resolution of 1-3 $\mu \mathrm{m}$. Areas within the primary, conjugate and mixed (primary + conjugate) strain regions of the $\mathrm{x}$-face were identified and mapped for their orientation, excess defect density and shear stress. The mesoscopic defect structure was concentrated in broad, somewhat irregular primary bands that lay nominally parallel to (111) in an almost periodic distribution with a period of about $30 \mu \mathrm{m}$. These primary bands were dominant even in the region of conjugate strain. There were also broad conjugate defect bands, almost precisely perpendicular to the primary bands, that tended to bridge primary bands and terminate at them. The residual shear stresses were large (ranging to well above $500 \mathrm{MPa}$ ) and strongly correlated with the primary shear bands; interband stresses were small. The maximum resolved shear stresses within the primary bands were oriented out of the plane of the bands, and, hence, could not recover the dislocation structure in the bands. The maximum resolved shear stresses in the interband regions lay predominantly in $\{111\}$ planes. The results are compared to the mesoscopic defect patterns found in $\mathrm{Cu}$ in etch pit studies done some decades ago, which also revealed a mesoscopic dislocation structure made up of orthogonal bands.
\end{abstract}

Keywords: 
AMS Subject Classification:

\section{Introduction}

Crystal plasticity is a heterogeneous process. The heterogeneity is ultimately due to the fact that crystals deform at the microscopic level through the motion of discrete dislocations on particular slip systems, and is exaggerated by the fact that these interact to create entanglements, deformation bands and cellular patterns. But the heterogeneity extends to the intermediate (mesoscopic) scale and even to the macroscopic level, through the appearance of irregular deformation patterns.

While the basic features of microscopic plasticity are reasonably well known, there are few (if any) welldocumented cases in which the deformation of a crystal has been characterized across the spectrum of length scales with sufficient detail to show how the various levels connect together. One reason is that such a characterization study is tedious and requires the sequential application of a number of different experimental apparatuses and techniques. A second reason is that, until recently, the detailed characterization of plastic deformation at the mesoscale and macroscale was not entirely practical. However, with the advent of such probative techniques as 'image correlation', synchrotron radiation, electron backscatter diffraction (EBSD) and focused ion beam (FIB) machining, it is rapidly becoming practical to map the deformation pattern at every length scale from individual dislocations to macroscopic strain.

There is an increasing need for this kind of "multiscale characterization". Recent developments in the computational modeling of crystal plasticity promise significant advances in the predictability of plastic deformation [1,2]. However, these models are inherently "multiscale"; the approach is a sequential treatment of dislocations and their interactions, groups of interacting dislocations, mesoscopic deformation patterns, and macroscopic plasticity, with the analysis at each level informing and constraining the treatment of the level above and below. The experimental verification and refinement of such models requires that there be comparably well-characterized experimental examples that are correspondingly "multiscale" in their coverage.

The research that is reported here is part of an effort to accomplish the multiscale characterization of a $\mathrm{Cu}$ single crystal that was oriented to deform in single slip, a nominally simple example of plastic deformation. The crystal was deformed in nearly uniaxial compression. The 'macroscopic' strain that was developed during the test, which is, for our purposes, the average superficial strain over areas greater than about $500 \mu \mathrm{m}$ in linear dimension, was measured and mapped continuously by 'image correlation'. After the sample had been strained in compression to a bit over 10\%, the 'mesoscopic' strain, the average strain over areas greater than $\sim 1 \mu \mathrm{m}$ in linear dimension, was measured and mapped by selected area diffraction in the focused white beam of a synchrotron light source. The residual stress and the defect density were measured simultaneously. The mechanical test device and the compression tests have been described elsewhere [3,4]. The present paper is mainly concerned with the results of selected area diffraction. Complementary EBSD and supplementary transmission electron microscopic studies are underway to complete the multiscale characterization, and will be reported elsewhere.

Single-crystal copper was chosen for this research as a prototype material whose deformation behavior has been extensively studied at the microscopic level [5-16]. While there has been little recent work on deformation patterns in the mesoscopic $(>20 \mu \mathrm{m})$ and macroscopic regimes, the mesoscopic dislocation 
patterns in $\mathrm{Cu}$ were characterized in some detail through etch-pit studies done by Livingston [17, 18] and Basinski [19] some time ago. These provide useful background for interpreting the results of the 'image correlation' and synchrotron studies reported here.

\section{Experimental procedure}

The sample used for this work was a copper single crystal, 99.99\% pure that was grown via the Bridgman technique by Accumet, Inc. The crystal was cut by wire electrical discharge machining (EDM) into a rectangular parallelepiped like the one illustrated in Fig. 1, with a $5.5 \mathrm{~mm}$ square base, a $15 \mathrm{~mm}$ height and rounded edges. The longitudinal faces of the sample were cut perpendicular to the [ $\overline{2} 920$ ] direction so that the sample would be oriented for single slip on the primary slip system, (111)[ $\overline{1} 01]$, when loaded in uniaxial compression. The lateral faces were cut perpendicular to [1 21 ] (the $\mathrm{x}$-face) and [ $\left.\begin{array}{lll}49 & 22 & \overline{5}\end{array}\right]$ (the $\mathrm{y}$-face), to create the geometry shown in the figure. Fig. 2 includes a stereographic projection relative to the longitudinal axis, and also contains a table of calculated Schmid factors for the common fcc slip systems when the sample is compressed in this orientation.

\subsection{Sample Preparation}

The sample was deformed in nominally uniaxial compression in the '6DOF' apparatus at the Lawrence Livermore National Laboratory (LLNL). This apparatus is illustrated in Fig. 3 and is described in refs. [20, 4, 21]. It was designed to allow motion in six independent modes so that uniaxial compression would be essentially unconstrained. In this device a nominal point load is applied to a half-sphere that serves as the upper platen on the specimen. The lower platen rests on a set of ball bearings on a plane base. The upper half-sphere allows displacement along the vertical z-axis and rotation about the $\mathrm{x}$ - and $\mathrm{y}$-axes (tilt in the $\mathrm{zx}$ and zy-planes), while the mobile base allows displacement in the $\mathrm{x}$ or $\mathrm{y}$ directions and rotation about $\mathrm{z}$ (twist in the xy basal plane). If the loading mechanism and bearings are frictionless, the deformation path is uniaxial compression. The extent to which that condition pertains in the actual tests described here is discussed in ref. [4] and in the following.

The macroscopic strain was measured and mapped using a GOM Aramis 3-D imaging system (acquired from Trillion Quality Systems). This device measures the local strain by locating the positions of a distribution of microdots placed on the surface of the specimen. The positions are recorded in a CCD camera, and successive images are compared to measure local displacements and calculate displacement gradients ('differential image correlation' [22]). In the present case, angled pairs of cameras are focused on two orthogonal surfaces of the specimen during the test [23]. The angled pair provides a stereo view that is used to measure microdot displacements both in and normal to the surface plane. The two camera sets provide simultaneous strain measurements on orthogonal surfaces.

Image correlation (IC) was used to follow the strain in real time during the compression test. The strain was calculated from the IC data as the symmetric part of the local displacement gradient. The data points represent the average strain over a $640 \mu \mathrm{m}$ square centered on the point position plotted. The data points form a grid with a $250 \mu \mathrm{m}$ interpoint spacing. Hence the computed and plotted strains are "moving averages" of the local strain. The overall strain of the sample was computed as an average of the local strains on the sample surfaces. The data were recorded both as a data set and as a movie. A succession of still images from the movie is shown in Fig. 4, which includes color-coded maps of the deformation on the $\mathrm{x}$ - and y-faces as a 
function of strain at a strain rate of $10^{-3} / \mathrm{sec}$. The test was terminated when the axial engineering strain reached $10.28 \%$. There is, of course, some relaxation when the sample is removed from the apparatus $(\sim 0.074 \%)$, which is negligible as a fraction of the macroscopic strain. Direct comparison of strain maps taken in the loaded and unloaded conditions show that they are virtually indistinguishable.

The samples that provided most of the data presented in the following were prepared as follows. After deformation, $1 \mathrm{~mm}$ thick sections were cut parallel to the $\mathrm{x}$ - and $\mathrm{y}$-surfaces of the specimen by electrodischarge machining (EDM) (Fig. 5). The surfaces of the sectioned samples (which match those left on the original crystal) were prepared for x-ray analysis by polishing flat with $0.5 \mu \mathrm{m}$ diamond. This sample preparation procedure was chosen so that we could examine surfaces that were near enough to the external surface that the macroscopic deformation maps could be assumed to apply, while being sufficiently below the surface to avoid any surface effects on the mesoscopic deformation patterns. (While it is possible for $\mathrm{Cu}$ crystals with very low dislocation densities to have slip distances of a millimetre or greater, this situation pertains, if at all, only to the earliest stages of our test.)

The fact that the characterized samples were prepared by EDM machining raised the possibility that the cutting operation had deformed the specimens and altered the results, even after the subsequent polishing. There were a number of reasons to believe that this did not happen to any significant degree. Most obviously, as we shall show, the mesoscopic deformation patterns on the cut specimens correspond closely to the macroscopic deformation patterns and show no apparent influence from the cutting, and regions of the cutand-polished specimen that were essentially undeformed by the macroscopic deformation show no evidence of deformation from the cutting. This result is not surprising. Our crystal was relatively hard to begin with, and was deformed to $10 \%$ strain prior to cutting, so we expected that any damage from the machining operation would be confined to the immediate surface and polished away. Nonetheless it was important to investigate the possibility that the results were compromised by the sample preparation technique.

To test the influence of the sample preparation two additional sets of samples were prepared and characterized. First, the opposite, uncut faces of the tested specimen (Fig. 5) were electropolished and mapped in the synchrotron. As will be discussed further below (Fig. 11), the results are essentially identical to those obtained from the cut-and-polished surfaces. Second, a second sample had been deformed in the same test device, but at an undesirably high strain rate. The surfaces of this sample were electropolished and examined in the x-ray source. Their mesoscopic deformation patterns were, also, essentially the same [24].

Finally, the tested specimen had not been characterized before its test. Given its unusually high compressive yield strength, there was some concern that it may have contained a high and patterned dislocation density that pre-existed the test. We, therefore, selected a second crystal that had been identically prepared, but not tested. The specimen surfaces were electropolished flat and mapped in the synchrotron. No evidence of a mesoscopic defect pattern appeared [24].

\section{$2.2 X$-ray Diffraction}

The x-ray diffraction analyses were done on the x-ray microdiffraction beamlines 7.3.3 and 12.3.2 of the Advanced Light Source at the Lawrence Berkeley National Laboratory (ALS/LBNL). Beamline 7.3.3 is well suited for these studies because of the high brilliance of its synchrotron source, the focusing optics that produce an ultrafine beam size, its "white beam" capabilities and the positioning stages that allow the beam to be swept with a micro-scale step size over a one square centimeter area. The energy range is 5-14 keV and, 
since the beam comes from a bending magnet, the energy distribution is continuous. In the tests described here a polychromatic (white) x-ray beam from a bending magnet source was focused to a spot size of approximately $0.8 \times 1.2$ micron full width at half-maximum $(\mathrm{VxH})$ by a pair of elliptically bent KirkpatrickBaez mirrors. The sample was set in a reflective geometry at 45 degrees to the incident beam. The diffracted $\mathrm{x}$-rays were collected with a large area MAR x-ray CCD detector (MAR133, collection area $13.3 \mathrm{~cm}$ diameter) that was positioned at an angle of 45 degrees approximately $3 \mathrm{~cm}$ from the specimen. An exposure time of 1 second was used for all experiments. Other beamline parameters were set to obtain high intensity reflections but avoiding peak saturation on the CCD. A Ge ORTEC solid-state detector coupled with a multichannel analyzer also permits the collection of fluorescence signals for elemental mapping. Further details of the beamline setup can be found elsewhere [25-27]. The x-ray microdiffraction program on beamline 7.3.3 has been recently moved onto the superconducting magnet source beamline 12.3.2 where additional measurements were performed. Apart from the source difference providing enhanced capabilities, the instrumentation used on 12.3.2 is essentially the same as on 7.3.3.

The areas that were scanned were chosen to provide meaningful data in the limited beam time available. As can be seen in the strain maps in Figs. 4 and 5, and will be discussed further below, the [1 21$]$ (x-face) of the deformed crystal contained several distinguishable regions with distinct deformation patterns. To locate these regions three platinum markers were placed on the sample surface. These are shown in Fig. 6. The markers could be found by $\mathrm{x}$-ray microfluorescence during $\mathrm{x}$-ray analysis and identified by their distinct shapes. The areas that were surveyed by the $\mathrm{x}$-ray scans reported here were located by reference to the arrowhead on the central platinum marker. The selected areas were scanned with a step size of 1-3 microns. The initial experiments used a step size of 3 microns to map three selected areas on the surface of the [1 21$]$ face of the crystal. A square with a $297 \mu \mathrm{m}$ edge was mapped in the central region of the crystal while squares of 180 $\mu \mathrm{m}$ size were scanned at selected sites in the upper and lower regions. In subsequent sessions, a $0.030 \times 9.592$ $\mathrm{mm}^{2}$ area down the center of the crystal was mapped with a 2-micron step size to clarify the differences between the 3 initial scans and analyze the transitions between visually different regions on the crystal surface. In addition, two $50 \mu \mathrm{m}$ x $50 \mu \mathrm{m}$ regions were scanned with a one micron step size to provide the best resolution in the top 'dead-zone' portion of the crystal and in the central region of the crystal. The final set of scans mapped additional areas in each of 3 distinct regions of the crystal with a $2 \mu \mathrm{m}$ step size.

The data taken in these diffraction experiments are Laue diffraction patterns that sample an area roughly equal to the diameter of the focused beam $(\sim 1 \mu \mathrm{m})$ to a depth that approximates the penetration depth of the beam $(\sim 30 \mu \mathrm{m})$. The diffraction patterns were analyzed with XMAS (X-ray Microdiffraction Analysis Software), which was written in-house for the beamline at the ALS [28, 29]. Laue patterns from unstrained silicon reference samples were used as reference patterns for the analysis of the deformed sample. The patterns from the unstrained reference samples are used in the XMAS software to calibrate geometric parameters of the beamline during each session.

The measured Laue diffraction patterns contain three types of data. First, the average overall orientation of the sampled volume is measured from the orientation of the diffraction pattern. This data was used to map the total rotation from the original orientation of the specimen, and the misorientation of adjacent volumes within the specimen. Second, the misorientation within the sampled volume is measured by the half-width of the diffraction peaks. The peak streaking is anisotropic. The long $(\mathrm{y}-)$ axis of the streaked peak is a consequence of the local curvature of the lattice. The curvature is ordinarily due a local excess density of the dislocations whose Burgers vectors accomplish the deformation and, hence, provides a measure of the 'geometrically necessary' dislocation density [30]. Third, the average deviatoric strain within the sampled volume is 
measured by the distortion of the diffraction pattern. The deviatoric stress tensor is computed from the strains using the anisotropic elastic constants of the material and was used to calculate the Von Mises (shear) stress, the maximum shear stress, and the resolved shear stresses on the preferred slip systems. Examples are presented in the following. Given the beamline geometric parameters and detector performances, the expected accuracy for copper samples in the orientation matrix is $\pm 0.01^{\circ}$, the accuracy in the strain tensors is $\pm 2 \times 10^{-4}$ and the stresses should be accurate to within $\pm 20-40 \mathrm{MPa}[25,29]$.

\section{$2.3 X$-ray data analysis}

The analysis software of the microdiffraction beamline, the X-ray Microdiffraction Analysis Software (XMAS), processes the raw diffraction data. This software package was developed for use at the Advanced Light Source at LBNL, and is similar (or identical) to that used in other advanced x-ray facilities. It is described in detail in refs. [31, 32, 28, 33]. The software automatically takes each pattern through image processing, peak indexing, strain refinement and stress determination steps using inputted crystal parameters and calibration to define the experimental geometry. The software incorporates display and analysis tools to manipulate the data and relate the individual patterns to one other.

2.3.1 Image processing. The image processing includes background removal and peak identification. The background intensity is removed via an algorithm that fits it with a smoothly varying function and subtracts it. Peak identification utilizes several parameters such as, peak detection threshold, peak fit type (peak fitting allows for subpixel resolution over the peak position), search box size and maximum allowed deviation between peak fit position and intensity-weighted centroid position. These parameters are optimized before automated indexing begins to find the largest number of peaks while minimizing errant points. In this work we typically used a 2-dimensional Lorenzian peak fit, a search box size of 11 pixels, a minimum peak width of 0.1 pixels, a maximum peak width of 20 pixels, and a maximum peak-to-centroid deviation of 5 pixels. The peak detection threshold varied depending on the overall intensity of the background in the Laue patterns and scales roughly with the intensity of the incoming x-ray beam. The peak intensities varied with the sample, beam intensity and the beamline characteristics. As expected, the diffraction peaks become more diffuse and less intense as the degree of deformation increases. Before applying the automatic analysis, the threshold value was optimized by hand for a few Laue patterns so that the peak identification process would consistently find the majority of peaks in the Laue pattern.

2.3.2 Indexing. The diffraction patterns are indexed using the geometry of the beamline and the nature of the diffracting crystal. The diffracting crystal is specified by its parameters, its Wyckoff positions and occupancies, and its elastic moduli. The geometry of the beamline was calibrated with Laue patterns from a reference sample, a (100) silicon wafer in this case. The calibration is done by indexing the reference pattern and performing a non-linear least squares refinement by minimizing the differences between the experimentally determined peak positions and the calculated ones, to determine the geometrical beamline parameters, including the sample-detector distance, the $\mathrm{x}$ - and $\mathrm{y}$-center CCD center positions, and the tilt angles of the detector with respect to the beam. A calibration from a silicon reference sample was determined good when the average differences between the measured and calculated peak positions are less than $0.01^{\circ}$.

The software calculates the scattering vectors associated with each peak found during peak fitting. The brightest reflections and their associated scattering vectors are compared against a reference list of scattering vectors calculated for the particular crystal structure. The indexing is completed by comparing triplets of 
angular matches between the experimental and theoretical lists of scattering vectors. The triplet that matches the largest number of reflections present in a frame is used for the indexation.

2.3.3 Computing the deviatoric strain and stress. The local value of the deviatoric strain is measured from the indexed white-beam diffraction pattern by calculating the differential angular displacements of the Laue spots. These correspond to changes in lattice angles and, therefore, measure the deviatoric strain. The dilatational component of the strain was not measured in this work, for two reasons. First, the measurement requires the exact wavelength of at least one of the Laue reflection, which, in turn, requires taking an energy scan at every single point of the large microdiffraction maps. This is a time consuming effort, which was not practical given the limited beam time available to us. Second, the dilatational component was not needed for the calculations we wished to make, the resolved shear stresses and the Von Mises shears that are reported in the results section.

An accurate calculation of the centroid positions of the Laue spots requires, of course, that the diffraction peaks not be truncated by limited band width in the incident white beam. In the present case there should be no appreciable truncation since the extent of the peaks is much smaller than the bandwidth of the white beam. A simple approximation for the energy extent of the streaks is to take the derivate of the Bragg equation. This equation expresses the energy extent of the streak in terms of the (measured) angular extent.

$$
\cot (\theta) \Delta \theta=\frac{\Delta E}{E}
$$

Where $\Delta E$ is the energy broadening of the diffracted peak caused by the divergence $\Delta \theta$. Choosing a few of the most severely streaked peaks from the Laue patterns, the extent of the streaks are on the order of 100-200eV whereas the continuous energy range from the bending magnet is approximately $9 \mathrm{keV}$. Therefore, the streaks are not significantly truncated.

The diffraction pattern measurements return the lattice parameter ratios (b/a, c/a, b/c) and the angles between the lattice parameters. The deviatoric strain tensor is computed from this data following the procedure outlined in [28]. The accuracy of the strain measurement is, of course, dependent on the number of peaks used in the calculations. Based on analysis in [33], the error in the strain measurement is less than $\pm 2 \times 10^{-4}$ for most sets of reflections analyzed in this work.

The deviatoric stress tensor is calculated from the deviatoric strain tensor using the reference values of the anisotropic elastic moduli. The resolved shear stress (RSS) can then be found for a given slip plane by calculating the tractions on that plane. The maximum resolved shear stress (MRSS) is the maximum RSS value calculated for all possible glide systems, which were taken to be the $\{111\}<110\rangle$ systems for fcc $\mathrm{Cu}$.

\subsection{Measuring the dislocation density}

The geometrically necessary, or excess, dislocation content (GND) can be calculated from the Cahn-Nye [35] relationship for relating lattice curvature to excess dislocation content:

$$
\rho_{G N D}=1 / R b,
$$


where $\mathrm{R}$ is the local radius of lattice curvature and $\mathrm{b}$ is the Burgers vector (the [110] spacing in the case of $\mathrm{Cu}$ ). The radius, $\mathrm{R}$, is computed on the assumption that the local lattice curvature contained within each Laue spot is reflected in the asymmetric peak broadening. XMAS returns the average value of the asymmetric broadening of all indexed peaks in a Laue image in degrees $(\psi)$, which is the angular turn over a chord equal to the beam size $(\delta)$. This value is calculated after taking out the isotropic and "instrumental" broadening (which are negligible compared to the streaking in the severely deformed regions). It follows that

$$
R=\delta /[2 \sin (\psi / 2)]
$$

which is substituted into (1) to obtain $\rho_{\mathrm{GND}}$. Given the subpixel peak fitting resolution of XMAS, the angle $(\psi)$ is determined to an accuracy of about $\pm 0.01^{\circ}$. For the calculation of $\rho_{\mathrm{GND}}$, this accuracy is reduced by the contribution of isotropic broadening to the peak width, a factor we have not been able to quantify precisely, but believe to be small, particularly for the regions of high dislocation density where the asymmetric broadening is pronounced.

\section{Results and Discussion}

\subsection{Macroscopic strain patterns}

The sample studied here was oriented for easy glide on the (111)[ $\overline{101}$ ] system when loaded in uniaxial compression and was tested in the 6DOF fixture at LLNL. The engineering stress-strain curve from the test is in Fig. 4. The crystal yielded at about $16 \mathrm{MPa}$. The crystal deformed in 'easy glide' to a strain of about 0.025 and afterwards hardened almost linearly to the final strain, $\sim 10.28 \%$, where the test was terminated. The stress-strain data from this and other tests is discussed in more detail in ref. [36]. The yield strength of this sample was high compared to that measured in other tests and reported by other investigators [36]. The reason appears to be that this crystal was tested in the as-cut condition, and residual defects from the original crystal growth and machining strengthened the crystal. However, the hardening behavior and deformation patterns in this crystal were qualitatively the same as those observed in annealed crystals with lower strength.

The pattern of deformation during the compression test is shown as a function of strain in the sequence of image correlation maps of the axial strain $\left(\varepsilon_{\mathrm{yy}}\right)$ in Fig. 4. These were taken from films made during the test. The interesting behavior occurs in the $x$-face (perpendicular to [ $\left.1 \begin{array}{ll}1 & 21\end{array}\right]$ ). During the 'easy glide' stage of deformation the strain develops in a band on the $\mathrm{x}$-face at $45^{\circ}$ to the compression axis (z-axis), and gradually intensifies. This deformation band parallels the trace of the (111) glide plane and will be referred to as the 'primary' band.

A well-defined 'conjugate' deformation band also forms, with an orientation $90^{\circ}$ from the primary. Like the primary band, the conjugate band is present from the beginning of the test [36], and is visually obvious in the strain map when the compressive strain reaches about $2 \%$ (Fig. 4). The crystallographic habit of this conjugate band also lies on a plane of maximum shear at $45^{\circ}$ from the compression axis; its habit is essentially parallel to $(10 \overline{1})$, which, significantly, is not a preferred slip plane for fcc copper. Both the primary and conjugate bands intensify as the strain increases, with the axial strain concentrated particularly in the central region of mixed deformation where the two bands cross. 
Fig. 4 also traces the development of deformation on the y-face as a function of strain. On this face the strain is concentrated in essentially parallel bands, perpendicular to the compressive axis. At low strain these are most pronounced in the lower area of the face, where the primary slip bands intersect this face. At higher strains horizontal deformation bands also decorate the upper area of the y-face. These bands correspond to intersections of the conjugate slip bands on the $\mathrm{x}$-face.

Fig. 7 presents maps of all the strains on the $\mathrm{x}$ - and $\mathrm{y}$-faces. On the $\mathrm{x}$-face, the strains $\varepsilon_{\mathrm{zz}}$ and $\varepsilon_{\mathrm{yy}}$ are pronounced. They have the same geometric pattern, but opposite signs (plastic deformation conserves volume and, therefore, tends to conserve area). The magnitude of the strain is slightly greater in the y-direction $\left(\varepsilon_{\mathrm{yy}}\right)$. There is, in addition, a local shear strain, $\varepsilon_{\mathrm{yz}}$, in the secondary deformation band on the x-face which has a maximum value of less than 0.01, with a slightly smaller local shear in the primary band (the shear map is a bit deceptive because of the colors used to code the strain). Local shears also appear at the upper left- and lower right-hand corners of the $\mathrm{x}$-face, which suggest that there was some slight frictional deformation from the platens of the test fixture at these two positions. These local shears appear in the last stages of deformation.

The stain field on the $y$-face is comparatively simple. The axial compression, $\varepsilon_{\mathrm{zz}}$, is the only significant strain. Its pattern and magnitude appear to be consistent with the constraints that the total compression should be nearly the same on the $\mathrm{x}$ - and $\mathrm{y}$-faces, and that volume should be conserved, with $\varepsilon_{\mathrm{yy}}$ slightly greater in magnitude than $\varepsilon_{\mathrm{zz}}$ on the $\mathrm{x}$-face. There is a slight enhancement of $\varepsilon_{\mathrm{zz}}$ at the upper and lower edges of the yface, and a slight indication of shear $\left(\varepsilon_{\mathrm{xz}}\right)$ on these edges.

\subsection{Mesoscopic defect patterns}

To characterize the mesoscopic defect patterns we used $\mathrm{x}$-ray microdiffraction to map selected areas of the $\mathrm{x}$ and y-surfaces. The scanned areas were selected to explore interesting features in the macroscopic strain maps.

3.2.1 Defect patterns on the $y$-face. We begin with a description of the defect patterns on the $y$-face, since these are much simpler than those on the $x$-face. Note that the traces of the preferred (111) slip plane are horizontal lines on the $y$-face, perpendicular to the z-axis of compression (Fig. 7), and that the preferred slip direction, [ $\overline{101}$ ], has no x-component in the y-face. It follows that slip in the preferred system causes an outof-plane deformation on the $\mathrm{y}$-face in bands oriented perpendicular to the $\mathrm{z}$-axis (the axis of compression). The slip system with the second largest Schmid factor is the ( $\overline{111})$ [101]. There is some evidence in Fig. 7 for the activation of this slip system in the y-face. The trace of this slip plane makes an angle of about $30^{\circ}$ to the horizontal in the $y$-face, and would cause slip in the $\mathrm{x}$-direction, transverse to the plane.

The residual defect patterns that are revealed by microdiffraction on the y-face are shown in Fig. 8 . The insert figure on the left hand side of Fig. 8 shows the surface of the specimen. The macroscopic strain map obtained from image correlation is superimposed. Note that, as illustrated in the upper figure, the surface scanned was the inner surface of the cut layer, so the strain map that appears is the mirror image of that shown in Fig. 4. The microdiffraction data is taken near the square indicated in the right center of the face, in the region where the strain is most severe. Five data sets are plotted on the right-hand side of the figure. 
The top figure (Fig. 8a) is the 'out-of-plane' orientation map that shows the tilt of the y-face. The tilt appears in broad, irregular bands with well-defined edges. The bands are roughly perpendicular to the axis of compression and are 10-40 $\mu \mathrm{m}$ in width. They are also roughly periodic with a spacing of 30-40 $\mu \mathrm{m}$. Morphologically, they resemble the heterogeneous bands of macroscopic strain on the surface of the specimen, and show that the heterogeneous pattern persists to much higher magnification. The roughly constant angle within the bands shows that the 'geometrically necessary' defects that cause the tilt are concentrated along the edges of the bands. Note that the tilt angle is small; it changes by only about $1.5^{\circ}$ over the whole area mapped. The fact that the bands are irregular, despite their clear edge definition may reflect participation by the secondary slip system, $(\overline{1} 11)[101]$. The ( $\overline{111})$ plane makes an angle of about $30^{\circ}$ to the horizontal in the figure and is, hence, roughly parallel to the sloped edges of some of the orientation bands.

The second figure (Fig. 8b) is a map of the in-plane orientation over the same area. The orientation is, again, heterogeneous and banded in the direction perpendicular to the compression axis. Compared to the out-ofplane orientation pattern, the in-plane orientation bands are less well defined, their boundaries are more diffuse and the total misorientation is smaller, only abut $0.5^{\circ}$. Since the primary slip system does not produce displacement in the $\mathrm{x}$-direction on this face, the in-plane misorientation bands must reflect the participation of secondary slip systems. This may explain the relative lack of definition of the bands.

Figs. 8c and 8d are local measures of misorientation. Fig. 8c maps the "intercell" misorientation between adjacent diffracted areas ("pixels' that are approximate squares $2 \mu \mathrm{m}$ on a side). Fig. 8d maps the "intracell" misorientation within the pixels, as reflected in the angular range of the long axes of the asymmetric diffraction spots. Since the lattice misorientation is accomplished by crystal defects, particularly 'geometrically necessary' dislocations, both are measures of the dislocation density. The intracell misorientation is the more direct measure since it is more local, and we have used it to define the excess dislocation density. A comparison of Figs. 8c and 8d shows that the two measures of misorientation have almost identical patterns, which indicates that both are measuring the same mesoscale average of the local excess dislocation density. Moreover, as expected, the bands of high dislocation density are located along the well-defined boundaries of the out-of-plane deformation bands (Fig. 8a). The excess dislocation density within the high-density bands is in the range $6 \times 10^{13}-8 \times 10^{13} \mathrm{~m}^{-2}$, and is roughly an order of magnitude above the background value, $\sim 1 \times 10^{13}$ inside the orientation bands.

The distribution of shear stresses was also extracted from the microdiffraction data. Fig. 8e is a pixel-by-pixel map of the maximum resolved shear stress over the scanned region. Note that the maximum shear stress exceeds $500 \mathrm{MPa}$, and is far above the critical resolved shear stress for easy glide in this crystal $(<10 \mathrm{MPa})$. Comparison with Fig. 8d shows that the bands of high shear stress coincide with the bands of high dislocation density. The high shear stress is likely associated with dislocation pile-ups and entanglements on the boundaries of the misorientation bands. There is, in fact, much more stress information contained in the microdiffraction data; the full deviatoric stress tensor can be calculated for each cell. Some of the additional data are presented below.

3.2.2 Defect patterns on the $x$-face. The slip vector of the primary slip system lies in the $x$-face of the crystal with the consequence that the heterogeneous defect patterns are most apparent in this face. The pattern is shown in the maps contained in Figs. 9-11. In each of these figures the insert figure on the righthand side locates three areas that were scanned. The three areas are 180-300 $\mu \mathrm{m}$ on a side and are sited, respectively, within the broad band of primary strain, near the center of the region of mixed primary and conjugate strain, and within the band of predominantly conjugate strain. Three data sets are mapped for each 
area: the in-plane orientation, the intercell misorientation and the intracell misorientation (which is used to calculate the excess dislocation density).

The three figures differ in the following ways. Figs. 9 and 10 are maps of the $\mathrm{x}$-face cut and polished from the primary sample. In Fig. 9 the maps in the primary and conjugate bands are taken from areas very close to the mixed (primary + conjugate) band, so Fig. 10 was prepared to show data from positions that are more clearly within the primary and conjugate bands. There are, however, discontinuities in the maps in Fig. 10 that are experimental artifacts caused by discontinuous steps in the sample stage. While these do not compromise the data, they make the maps more difficult to read. The maps shown in Fig. 11 were taken from the opposite $\mathrm{x}$-face of the tested specimen. The macroscopic strain on this face was mapped before and after the test, but not during loading. The three sites for the mesoscopic maps were selected from by examining the strain map after deformation (this is the map shown in the figure). This figure was prepared and included to show that the data are not obviously affected by the method of sample preparation. Aside from an overall curvature in the deformation pattern in the mixed band, the patterns are visually the same. As noted above, with the exception of this figure, all of the data that is shown is taken from the cut-and-polished surface.

While the macroscopic strain patterns differ dramatically from the primary to the overlap to the conjugate regions, the mesoscopic patterns are surprisingly similar. In all three regions the in-plane orientation map is cross-hatched by broad bands with defined boundaries. The bands lie at roughly $\pm 45^{\circ}$ to the $\mathrm{z}$-axis (the axis of compression). The most pronounced bands lie roughly parallel to the (111) primary slip plane. The crosshatched bands are on the conjugate shear plane, which does not parallel any close-packed plane of the fcc crystal, and appears to represent the 'polygonal slip' that was observed in the etch-pit studies of Livingston $[17,18]$ and Basinski [19]. The maximum in-plane misorientation in the regions mapped varied from $\sim 1^{\circ}$ to $\sim 2.5^{\circ}$.

The intercell misorientation and the excess dislocation density (intracell misorientation) maps are presented in Figs. 9-11. If the cell size is sufficiently small that the excess dislocation density is uniform across a cell, and if different measures of misorientation are consistent, then the two measures of the misorientation should be nearly the same. Fig. 12 presents an additional series of mesoscopic maps in which the two misorientation maps have been superimposed to show their virtual coincidence. Fig. 12 also includes superimposed maps over three long strips, one taken in the primary deformation band, two in the mixed region of primary and conjugate shear.

As expected, the excess dislocation density is concentrated in narrow bands that outline the in-plane misorientation bands. The mesoscopic defect distributions in the three regions have a surprisingly similar appearance given the very different macroscopic strain patterns. In all three regions the most pronounced defect bands are roughly parallel to the primary (111) slip plane and extend across the field of view. The primary bands are quasi-periodic with a separation of about $30 \mu \mathrm{m}$ (the separation is a bit larger in the conjugate band). They are somewhat irregular in shape, a feature that suggests the participation of secondary slip planes, particularly the ( $\overline{1} 11)$, whose trace is approximately $67^{\circ}$ from the $\mathrm{z}$-axis, and the $(11 \overline{1})$, at about $22^{\circ}$ from the z-axis. The perpendicular (conjugate) dislocation bands are more diffuse and less extended. They are present in all three regions, but are more common and pronounced in the mixed and the conjugate bands. They often connect primary bands and terminate on them. Fig. 13 includes Fourier transforms of a number of dislocation density distributions from each of the three regions, and shows that the primary and conjugate bands are almost precisely perpendicular to one another. The measured excess dislocation densities 
in these high-density bands range up to about $5 \times 10^{13} \mathrm{~m}^{-2}$, against a background density that is a bit over $10^{12}$ $\mathrm{m}^{-2}$.

Finally, Fig. 14 shows out-of-plane orientation maps taken over a number of areas, and along a strip that extends from the 'dead' region at the top of the crystal down to the bottom of the region of mixed deformation. Since the primary slip is in the x-plane, the out-of-plane misorientation must reflect slip on secondary systems. As the figure shows, the out-of-plane orientation maps are subdivided into bands that roughly parallel the primary deformation bands. This observation remains true even for scans taken well within the region of conjugate slip. The bands are, again, quasi-periodic with a period of about $30 \mu \mathrm{m}$. The misorientation across their boundaries is no more than about $0.5^{\circ}$. The long scan down the center of the sample face shows that the crystal is very slightly bowed, possibly due to a small reaction at the platens during the later stages of the test.

\subsection{Mesoscopic stress patterns}

One of the attractive capabilities of microdiffraction as a characterization tool is its ability to measure the local value of the internal stress. In fact, the microdiffraction data reveals the full deviatoric stress tensor. In the following we discuss three measures of the stress: the in-plane shear stress, $\sigma_{\mathrm{yz}}$, in the x-surface of the sample, the overall shear stress, as measured by the Von Mises stress, and the maximum resolved shear stress available to drive dislocation glide.

Fig. 15 includes a plot of the in-plane shear stress, $\sigma_{\mathrm{yz}}$, in the $\mathrm{x}$-face of the crystal. The shear stress is measured at intervals of $20 \mu \mathrm{m}$ along the line indicated in the figure, which is plotted on the background map of the compressive strain. The mean value of the stress is very close to zero, as expected. Note the high values of the residual shear stresses. These reach several hundred MPa, which is far above the macroscopic shear stress applied during the compression test. As shown in Fig. 15(b) the values of the residual shear stress have a roughly Gaussian distribution with a standard deviation of about $100 \mathrm{MPa}$. The distribution of residual shear stresses is roughly the same over the whole length of the crystal, despite significant differences in the magnitude and direction of the macroscopic strain from one region of the crystal to another. There is, however, a tendency for the highest residual stresses to be found in the transition regions where the macroscopic strain gradients have their largest values.

Fig. 16 shows the distribution of the Von Mises stress over the x-face. The left-hand side of the figure exhibits maps of the local distribution of stress over small, selected areas, 50-180 $\mu \mathrm{m}$ on the edge. The righthand side shows the distribution of stress over $30 \times 800 \mu \mathrm{m}^{2}$ strips taken in the three distinct regions of macroscopic strain.

The distribution of the Von Mises stress has three striking features. First, the Von Mises stress is surprisingly large. It exceeds $1 \mathrm{GPa}$ in many locations within the crystal, far above the maximum value of the shear applied during the test.

Second, the local areas of very high stress, which are numerous, are organized into well-defined bands. The most pronounced of these bands parallel the primary bands of high dislocation density that were identified in the previous section. To emphasize this we have re-plotted data from Fig. 16 in Fig. 17, where they are placed alongside the corresponding plots of the excess dislocation density. As this figure makes clear, the high shear stress is within or in the immediate vicinity of the regions of high dislocation density; the high 
shear stress appears to be caused by dislocation activity and entanglements in the primary shear bands. High shear stresses are also found in bands along the conjugate shear directions, perpendicular to the primary bands. The stresses in these conjugate bands are, in general, less intense and more diffusely distributed than those in the primary bands. The association between the conjugate stress bands and the conjugate bands of high dislocation density is also a bit less clear.

Third, the bands of high residual stress tend to parallel the bands of primary slip, even in areas where conjugate slip dominates the macroscopic strain. Bands of stress do appear on conjugate planes with some frequency in the central (primary plus conjugate strain) and lower right (conjugate strain) regions, and are particularly common in the conjugate strain region. However, these are less intense and more diffuse than the bands in the primary direction. Moreover, particularly as shown by the maps in Fig. 16, they tend to connect primary bands and terminate on them, as if they were secondary features.

Finally, Figs. 18-20 illustrate the pattern of the maximum resolved shear stress (MRSS). The MRSS is the highest value of stress resolved on the 12 possible $\{111\} /<110\rangle$ dislocation glide systems in the fcc crystal. Fig. 18 presents maps of the MRSS over selected areas in the three distinct regions of macroscopic deformation. The MRSS patterns are very much like those of the Von Mises stress. The MRSS reaches high values $(\sim 600 \mathrm{MPa})$ and the high stresses are located predominantly in bands along the directions of primary shear. The MRSS is also high in conjugate bands oriented perpendicular to the primary bands. These bands tend to be weaker and more diffuse. They tend to connect the primary bands and terminate on them. The MRSS in the broad regions between defect bands is small, of the order of $60 \mathrm{MPa}$, which is close to the intracell stress that was recently measured by Levine, et al [37] in more severely deformed $\mathrm{Cu}$ that had developed a well-defined cell structure.

Fig. 18 includes maps that show the direction of the maximum resolved shear stress. Again the pattern follows the pattern of primary and conjugate bands. Interestingly, the highest stresses, which lie in the primary slip bands parallel to (111), are focused in directions, predominately [101], that do not lie in the (111) plane and would be difficult to relax by slip in (111). The maximum shear stress between bands tends to be

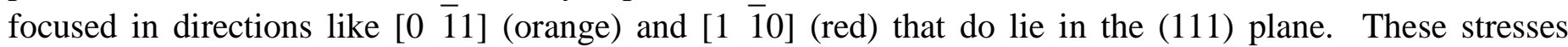
apparently could be relaxed by slip in (111), but the residual stress is very small; the stress has already been relaxed.

The pattern of stress concentration and relaxation is explored further in Fig. 20, on which we have superimposed plots of the MRSS and the excess dislocation density. The primary bands of high dislocation density superimpose almost precisely on the bands of MRSS. Interestingly, however, the conjugate bands of high excess dislocation density are not bands of high MRSS, even in the region where the macroscopic strain is dominated by conjugate slip. It appears that the primary dislocation bands tend to accumulate dislocations of the same sign, creating high residual stresses that cannot be relieved by glide in the slip plane. The conjugate dislocation bands predominate in regions of low stress, suggesting that these bands were formed in the process of relaxing shear stresses that could be relieved by primary slip.

\section{Discussion}

As discussed in the introduction, the work reported here had three purposes: to demonstrate a technique for multiscale characterization, to provide a characterized example of bulk deformation for comparison with simulation results and to clarify the mechanisms of plasticity in single crystal copper. The characterization 
studies done here stopped at the mesoscopic scale. The microscopic characterization of the dislocation processes that underlie these deformation patterns is in progress. We also note that the mesoscopic characterization was done on a single sample at a single macroscopic strain, about $10 \%$ axial compression.

Even in this simple case, nominally uniaxial compression of an fcc $\mathrm{Cu}$ crystal oriented for single slip, deformation at all spatial scales involves heterogeneities that need to be taken into account if the overall deformation is to be understood. Moreover, the deformation patterns include features that are not obviously contained in the most widely accepted models of dislocation plasticity. Unanticipated patterns were found at both the macroscopic and mesoscopic levels.

\subsection{Macroscopic deformation patterns}

The macroscopic strain field (the strain over areas of dimension greater than about $100 \mu \mathrm{m}$ for our purposes) is heterogeneous, particularly over the $\mathrm{x}$-face, and divides this face into several distinct areas with characteristic features. As expected, the strain field includes a broad, well-defined band of shear parallel to the (111) slip plane that contains the most highly stressed slip system (the highest Schmid factor). However, this shear is accompanied by a well-defined band of conjugate shear with a nearly ( $\overline{101})$ habit that is almost exactly perpendicular to the (111) primary band. By symmetry, this conjugate plane experiences a shear stress equal to that on (111) (at least initially), but contains none of the preferred slip systems of fcc, and, hence, shear on this habit plane does not conform to the Schmid model.

But at the same time that the deformation is more complex than anticipated, it remains relatively simple. The macroscopic strain field is not, at least superficially, a mixture of the many possible elementary strains. To a good first approximation, it is a simple superposition of two broad shear bands, one parallel to the slip plane that is expected from the Schmid model, and a second on the conjugate plane of maximum shear stress, as might be predicted on the basis of a macroscopic 'slip-line' model that ignored the crystallographic detail.

\subsection{Mesoscopic defect patterns}

Because the macroscopic strain had been mapped, it was possible to select areas for mesoscopic characterization that sampled regions with different macroscopic behavior. We particularly explored areas within the primary, conjugate and overlapped strained regions on the x-face of the crystal. The selected area diffraction studies reveal stresses, rotations and excess dislocation densities at a resolution down to about 1 $\mu \mathrm{m}$.

The heterogeneity and complexity of the deformation pattern increases at the mesoscale, but, interestingly, they do not provide an obvious explanation for the conjugate shear bands that appear on the macroscale. The most striking feature in all three regions is a almost periodic pattern of thick, wavy bands that generally parallel the primary (111) slip plane and are repeated about every $30 \mu \mathrm{m}$. Since these are the bands that are expected to carry the primary shear deformation, they should be present and dominant in the primary and mixed deformation regions. The (111) orientation of these bands is consistent with primary slip in the (111)[ 101$]$ slip system that has the maximum Schmid factor (Fig. 2). The waviness in the boundaries of the bands suggests some participation by secondary slip in the ( 111$)$ and $(11 \overline{1})$ planes, as described in the previous section. 
The primary deformation bands are connected or crossed by conjugate bands of defects that are relatively diffuse in appearance, but have an almost perpendicular orientation. These conjugate bands sometimes cross the primary bands to form a true cross-hatched pattern, but more commonly connect the extended, primary bands with short 'bridges' that terminate at both ends. This pattern suggests that the conjugate bands form after the primary bands and accommodate to them. The conjugate bands are present in all three regions, but are particularly common in the region of conjugate strain.

A mesoscopic defect structure composed of primary bands connected by secondary bands on perpendicular planes is a known pattern in deformed $\mathrm{Cu}$ that was first detected in the etch pit studies done by Livingston $[17,18]$ and Basinski [19] some fifty years ago. They studied single crystal $\mathrm{Cu}$ deformed in tension and bending at room temperature and at $4.2 \mathrm{~K}$, and observed a mesoscopic structure described as "polygonized glide' in which primary bands of tangled dislocations roughly parallel to the preferred slip plane were joined by perpendicular, relatively diffuse secondary bands. Insofar as could be inferred from the character of the etch pits, both bands contained a strong preponderance of dislocations of the same sign, which would lead to rotations across the defect bands that are consistent with the observations made here.

While the defect patterns in the 'primary' and 'mixed' regions are plausible and consistent with Livingston's 'polygonized glide', it is difficult to explain the retention of this structure in the region of conjugate shear. Shear by the conjugate bands is certainly plausible. Even if these are made up of the same dislocations as the primary bands, as Livingston proposed, a perpendicular array of them would create a tilt boundary that is, in effect, a plane of conjugate shear. But this observation does not explain why a macroscopic band of conjugate strain should have a structure that is dominated by extended bands of, apparently, primary shear.

Given that the understanding of the conjugate shear will have to wait more detailed studies, such as the TEM studies that are now underway, three possibilities suggest themselves. First, the dislocation density that is revealed by the selected area diffraction done here is an excess dislocation density that produces a local rotation. It remains possible that much of the macroscopic strain is due to a balanced dislocation distribution that cannot be inferred from the microrotation pattern. We doubt this explanation for two reasons. First, the Livingston-Basinski results suggest that it is not true. They found perpendicular, conjugate deformation bands that are, essentially, low-angle tilt boundaries made up of dislocations of like character. Second, the residual stress pattern almost precisely duplicates the measured pattern of the excess dislocation density, suggesting that this is the dominant residual dislocation density.

Second, the dislocations responsible for the conjugate strain may be so diffuse in their distribution that they contribute primarily to the diffuse background dislocation density rather than to the apparent defect bands.

Third, and, in our opinion, most likely, the detailed evolution of the defect distribution in the conjugate region may be such that it produces a rather different strain on the way to a superficially similar pattern. We note that the mesoscopic defect pattern shows where defects are, while the strain is a consequence of where they have been. If this is the case the polygonal dislocation structure (the diffuse conjugate bands) are the residue left from the formation and subsequent relaxation of the conjugate strain rather than its mechanism. 


\subsection{Mesoscopic stress patterns}

While there is prior work on the mesoscopic defect patterns in deformed $\mathrm{Cu}$, the present work is, to our knowledge, the first to map the mesoscopic distribution of stress. The results have several interesting features.

First, the magnitudes of the residual shear stresses are surprisingly large (Figs. 15-18). Whether the shear stress is measured by the in-plane shear $\left(\sigma_{\mathrm{yz}}\right)$, the maximum resolved shear stress or the Von Mises stress, the shear stress reaches values of 500-1000 MPa, which is much higher than the nominal shear strength of the crystal. These high stresses are concentrated in the primary shear bands and, to a lesser extent, the conjugate shear bands (Figs. 17, 18, 20). The fact that the maximum stresses appear to be in the defect bands, rather than adjacent to them, suggests that these high stresses are due to defect orientations and interactions that prevent their relaxation.

Second, and consistent with this, the maximum shear stresses in the highly stressed, primary defect bands are in crystallographic directions that do not lie in the (111) plane of primary slip (Fig. 19). It follows that these stresses cannot be relaxed by primary slip, a fact that may play a strong role in their preservation in the relaxed crystal. On the other hand, the stresses in the regions between the primary shear bands are in the (111) glide plane (though not in the [ $\overline{101}$ ] direction). Stresses in this plane should promote relaxations that contribute to the polygonization of the dislocation in the spaces between the primary shear bands.

Third, and also consistently, a superposition of the maximum resolved shear stress and the dislocation density (Fig. 20) shows both the strong correlation between high shear stress and the primary defect bands, and the much weaker correspondence between high shear stress and the conjugate bands. The relatively frequent observation of conjugate defect bands in regions of low residual stress suggests that the conjugate defect bands tend to form, or deform so as to relax the local stresses. The residual stresses in the relaxed material between bands is near $60 \mathrm{MPa}$, close to the value measured by Levine, et al. [37] within a well-developed dislocation cell in severely deformed $\mathrm{Cu}$.

\section{Summary and Conclusions}

We draw the following conclusions from this work.

1. It is practical and useful to employ 'multiscale characterization' with modern tools to clarify the nature and interaction of the heterogeneous deformation patterns and mechanisms that operate at different length scales. In particular, the combination of image correlation techniques to map the macroscopic strain and synchrotron (or, perhaps alternatively, EBSD) techniques provides detailed information on mesoscopic defect patterns.

2. In the particular case studied here, a copper single crystal oriented for single slip in the (111)[ $\overline{101}$ ] slip system and tested to $\sim 10 \%$ strain in nominally uniaxial compression, the macroscopic strain produced an inhomogeneous pattern of broad, crossed shear bands in the x-face of the crystal, which was oriented perpendicular to [ $\left.\begin{array}{ll}1 & \overline{2} \\ 1\end{array}\right]$ and contained the [ $\overline{101}$ ] direction of the preferred slip system. One, the primary band, lay parallel to (111). The second, the 'conjugate' band, had a nearly perpendicular ( $\overline{101}$ ) habit, even though the ( $\overline{101}$ ) plane contains no common slip plane of the fcc $\mathrm{Cu}$ crystal. The two bands divided the crystal into distinct primary, conjugate and mixed (crossed primary and conjugate) regions. 
3. Despite their macroscopic differences, the mesoscopic substructure within the three regions is surprisingly similar. As determined by selected area synchrotron diffraction with a resolution of 1-3 $\mu \mathrm{m}$, the dominant feature in all three regions is a quasi-periodic pattern of broad, somewhat irregular primary bands that lie nominally parallel to (111) with a period of about $30 \mu \mathrm{m}$. There are also broad conjugate defect bands, almost precisely perpendicular to the primary bands that tend to bridge primary bands and terminate at them. These are more common and pronounced in the region of conjugate strain.

4. The measured residual shear stresses are large (ranging to well above $500 \mathrm{MPa}$ ) and strongly correlated with the primary shear bands. The direction of maximum stress in the primary bands was oriented out of the (111) glide plane, and, hence, is not able to accomplish relaxation by shear in this plane. The maximum shear stresses between the primary defect bands are oriented in the (111) planes, which is consistent with the apparent relaxation and low residual stress in the interband material. While some of the conjugate defect bands have moderate to high shear stresses, most are almost devoid of shear stress, suggesting that the conjugate bands acted to relax the internal stress.

5. The results are generally consistent with the mesoscopic defect patterns found in $\mathrm{Cu}$ in etch pit studies done some decades ago [17-19]. In this case the primary bands were identified as narrow bands that contain predominantly dislocations of the same sign, connected by perpendicular "polygonization" bands that are also made up of like dislocations.

The results are being extended by high resolution TEM analyses, with particular emphasis on such puzzling questions as the nature of the conjugate bands and the mechanism that makes the macroscopic conjugate strain consistent with a mesoscopic pattern that is dominated by defect bands parallel to the plane of the primary macroscopic shear.

\section{Acknowledgements}

This work was performed under the auspices of the U.S. Department of Energy by University of California, Lawrence Livermore National Laboratory under Contract W-7405-Eng-48. The Advanced light Source is supported by the Director, Office of Science, Office of Basic Energy Sciences, of the U.S. Department of Energy under Contract No. DE-AC02-05CH11231 at Lawrence Berkeley National Laboratory.

\section{References}

[1] B. Devincre and L.P. Kubin, Mater. Sci. Eng. A 234 (1997), p. 8.

[2] W. Cai, V.V. Bulatov, T.G. Pierce, M. Hiratani, M. Rhee, M. Bartelt and T.M., Massively-Parallel Dislocation Dynamics Simulations, in Solid Mechanics and Its Applications, H. Kitagawa and Y. Shibutani, eds., Kluwer Academic Publisher, New York, 2004.

[3] D.H. Lassila, M.M. LeBlanc and G.J. Kay, Trans. ASME J. Eng. Mater. Tech. 124 (2002), p. 290.

[4] D.H. Lassila, M.M. LeBlanc and J.N. Florando, Met. Trans. 38 (2006), p. 2024.

[5] C.R. Cupp and B. Chalmers, Acta Met. 2 (1954), p. 803.

[6] E.N.D. Andrade, Proc. R. Soc. Lond. Ser. A Math. Phys. Eng. Sci. 240 (1957), p. 304.

[7] F.W. Young, J. Appl. Phys. 32 (1961), p. 192.

[8] J.W. Steeds, Acta Crystallogr. Sect. A 16 (1963), p. 108. 
[9] J.W. Steeds, Proc. R. Soc. Lond. Ser. A Math. Phys. Eng. Sci. 292 (1966), p. 343.

[10] M.R. Staker and D.L. Holt, Acta Met. 20 (1972), p. 569.

[11] T. Ungar, H. Mughrabi and M. Wilkens, Acta Met. 30 (1982), p. 1861.

[12] Q. Liu and N. Hansen, Phys. Stat. Sol. A 149 (1995), p. 187.

[13] Z.S. Basinski and S. J. Basinski, Phil. Mag. 21 (2004), p. 213.

[14] H. Mecking and G. Bulian, Acta Met., 24 (1976), p. 249.

[15] R.W. Cahn, J. Inst. Met. 79 (1951), p. 129.

[16] R.W.K. Honeycombe, J. Inst. Met. 80 (1951), p. 45.

[17] J.D. Livingston, J. Appl. Phys. 31 (1960), p. 1071.

[18] J.D. Livingston, Acta Met. 10 (1962), p. 229.

[19] Z.S. Basinski and S.J. Basinski, Phil. Mag. 9 (1964), p. 51.

[20] D.H. Lassila, M.M. LeBlanc and M. Rhee, Proc. Sympos. MRS 779 (2003).

[21] M.M. LeBlanc and D.H. Lassila, Exp. Tech. 20 (1996), p. 21.

[22] J.W. Dally and W.F. Riley, Experimental Stress Analysis, College House Enterprises, LLC, Knoxville, 2005.

[23] M.M. LeBlanc, J.N. Florando, D.H. Lassila, T. Schmidt and J. Tyson, Exp. Tech. 30 (2006), p. 33.

[24] K.R. Magid, The Hierarchical Characterization of Deformation Heterogeneities in Compressed Metal Single Crystals, Ph.D. Dissertation, University of California, Berkeley, 2007.

[25] N. Tamura, A.A. MacDowell, R. Spolenak, B.C. Valek, J.C. Bravman, W.L. Brown, R.S. Celestre, H.A. Padmore, B.W. Batterman and J.R. Patel, J. Synchr. Rad. 10 (2003), p. 137.

[26] N. Tamura, R.S. Celestre, A.A. MacDowell, H.A. Padmore, R. Spolenak, B.C. Valek, N.M. Chang, A. Manceau and J.R. Patel, Rev. Sci. Instrum. 73 (2002), p. 1369.

[27] N. Tamura, A.A. MacDowell, R.S. Celestre, H.A. Padmore, B. Valek, J.C. Bravman, R. Spolenak, W.L. Brown, T. Marieb, H. Fujimoto, B.W. Batterman and J.R. Patel, Appl. Phys. Lett. 80 (2002), p. 3724.

[28] J.S. Chung and G.E. Ice, J. Appl. Phys. 86 (1999), p. 5249.

[29] N. Tamura, H.A. Padmore and J.R. Patel, Mater. Sci. Eng. A 399 (2005), p. 92.

[30] J.F. Nye, Acta Met. 1 (1953), p. 153.

[31] J.S. Chung, N. Tamura, G.E. Ice, B.C. Larson, J.D. Budai and W.P. Lowe, Proc. Sympos. MRS 563 (1999), p. 169.

[32] N. Tamura, J.S. Chung, G.E. Ice, B.C. Larson, J.D. Budai, J.Z. Tischler, M. Yoon, E.L. Williams and W. Lowe, Proc. Sympos. MRS 1999, p. 175.

[33] B. Valek, X-ray Microdiffraction Studies of Mechanical Behavior and Electromigration in Thin Film Structures, Ph.D. Dissertation, Stanford University, 2003.

[34] D.P. Field, Personal communication, 2007.

[35] J.F. Nye, Acta Met. 1 (1953), p. 153.

[36] J. Florando, M.M. LeBlanc and D.H. Lassila, Scripta Mater. 57 (2007), p. 537.

[37] L.E. Levine, B.C. Larson, W. Yang, M.E. Kassner, J.Z. Tischler, M.A. Delos-Reyes, R.J. Fields and W.J. Liu, Nat. Mater. 5 (2006), p. 619. 


\section{Figures}

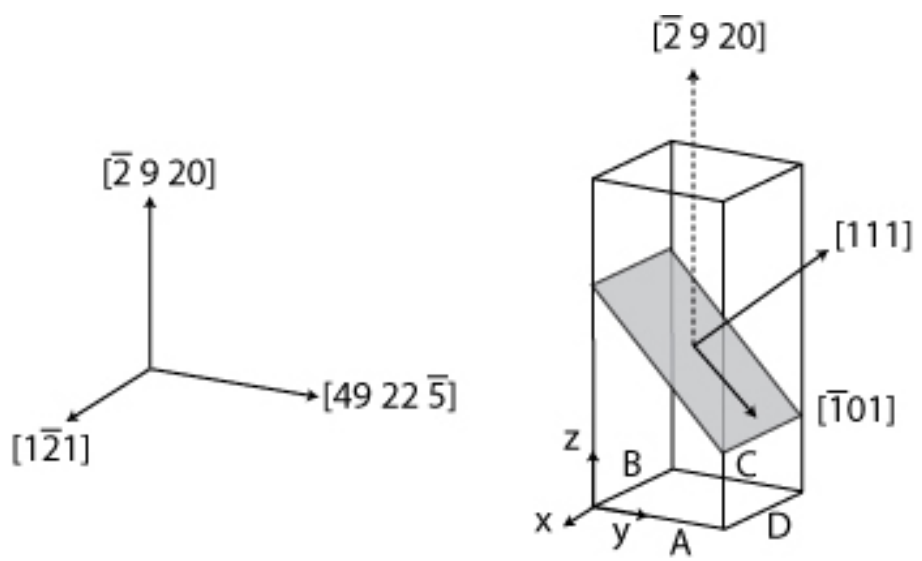

Fig. 1: The shape and orientation of the $\mathrm{Cu}$ test specimen. 


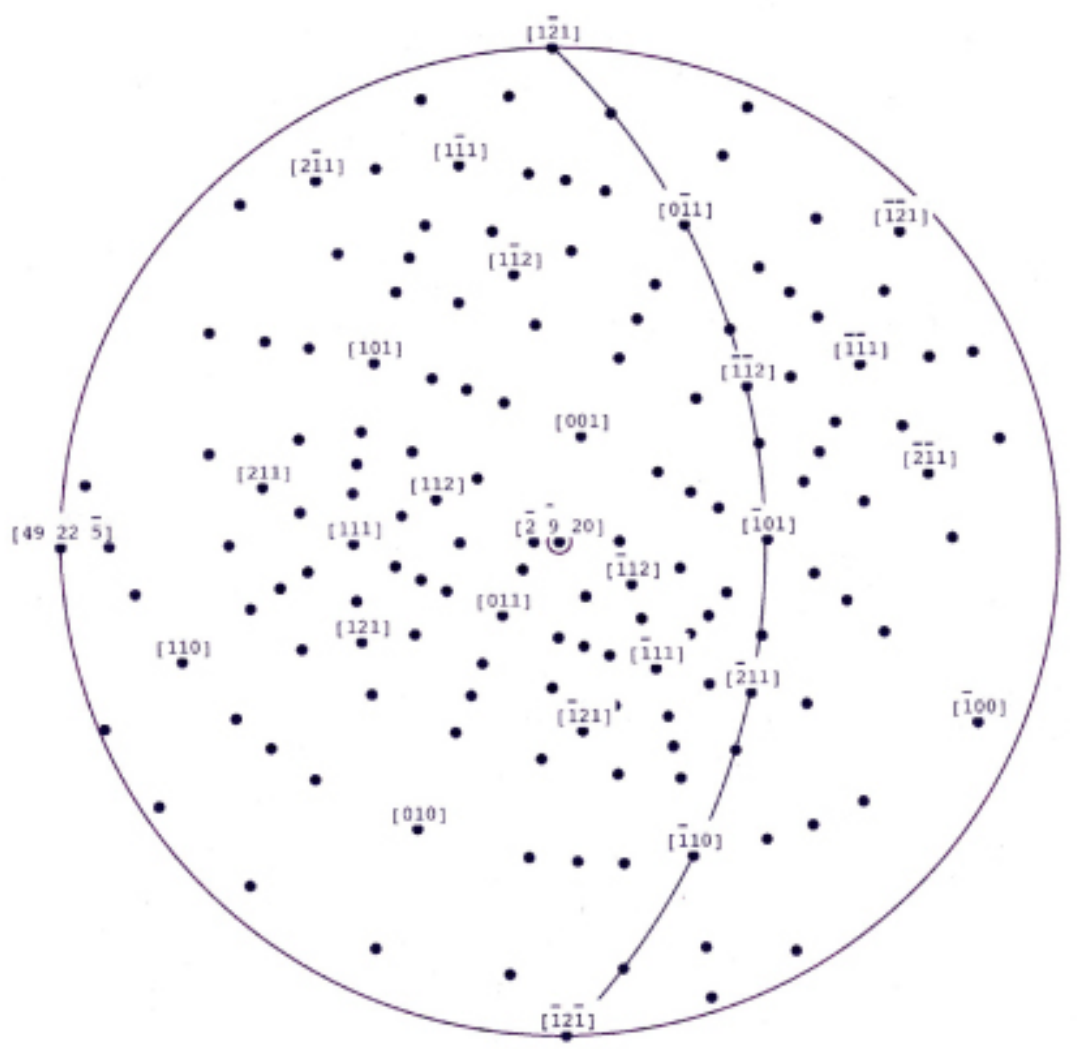

\begin{tabular}{cc} 
Slip System & Schmid Factor \\
\hline (111),[-101] & 0.50 \\
$(-111),[101]$ & 0.47 \\
$(1-11),[011]$ & 0.32 \\
$(-111),[0-11]$ & 0.29 \\
$(111),[1-10]$ & 0.25 \\
$(111),[0-11]$ & 0.25 \\
$(1-11),[-101]$ & 0.24 \\
$(-111),[110]$ & 0.18 \\
$(-1-11),[011]$ & 0.13 \\
$(-1-11),[101]$ & 0.079 \\
$(1-11),[[10]$ & 0.077 \\
$(-1-11),[-110]$ & 0.048
\end{tabular}

Fig. 2: Stereographic projection and tabulated Schmid factors for the crystal orientation shown in Fig. 1. 


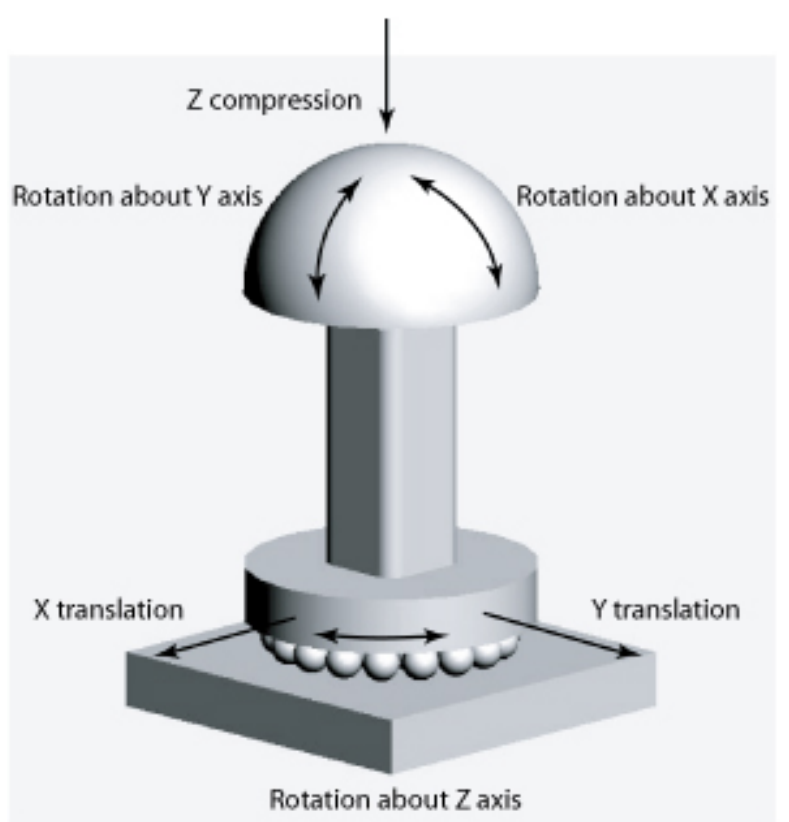

Fig. 3: Schematic of a compression test in the "6DOF" apparatus [1,2]. The objective is to allow unconstrained shape change. The six allowed deformation modes are translation in $\mathrm{z}$ (compression), translation in $\mathrm{x}$ or $\mathrm{y}$ (translation of the basal platen), rotation about $\mathrm{x}$ or $\mathrm{y}$ (tilt of the hemispherical upper platen) and rotation about $\mathrm{z}$ (rotation of the basal platen). 


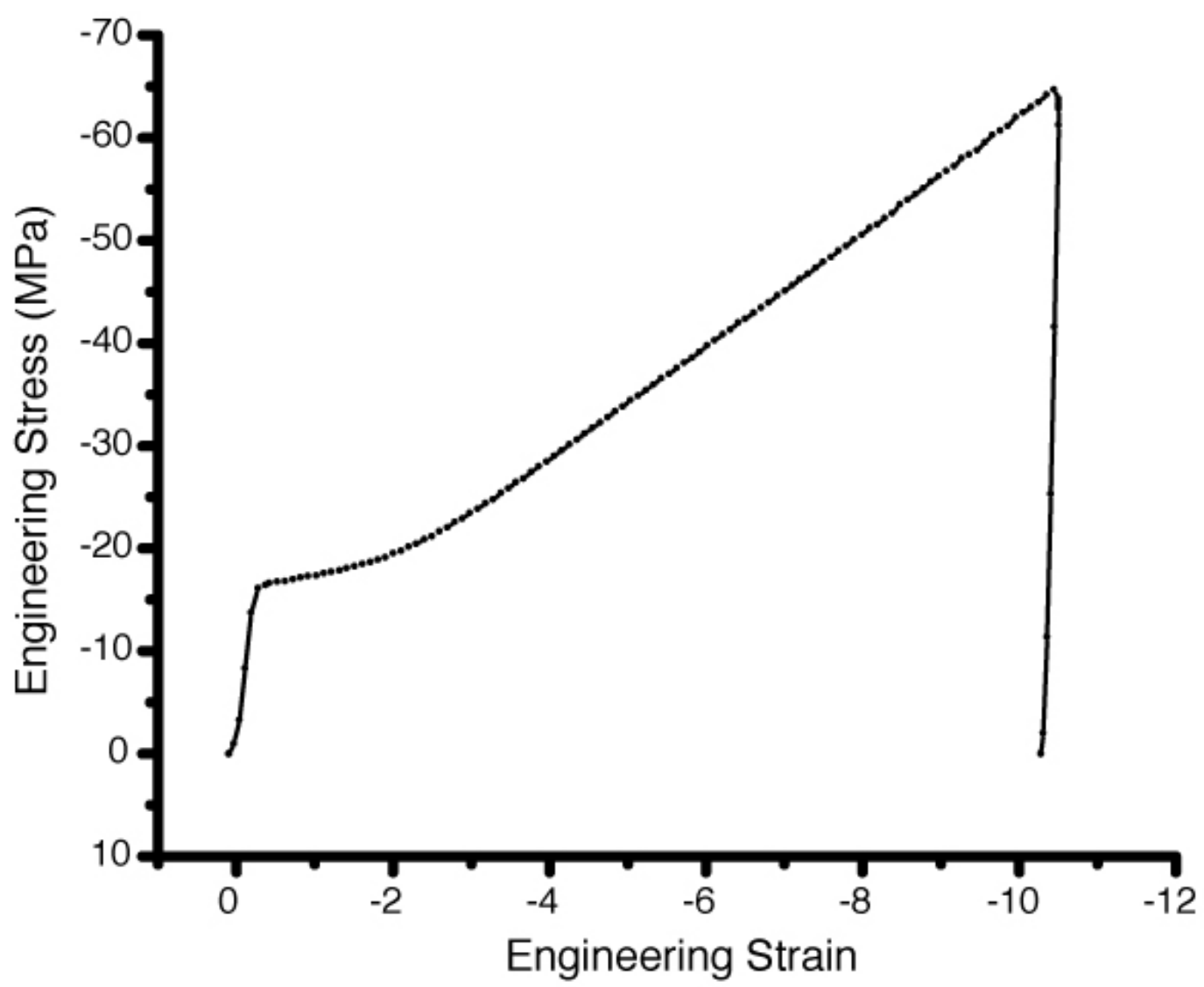

\section{Overall Axial}

Eng. Strain (\%)

$\mathrm{x}$-Face

[1 $1-2$ 1]
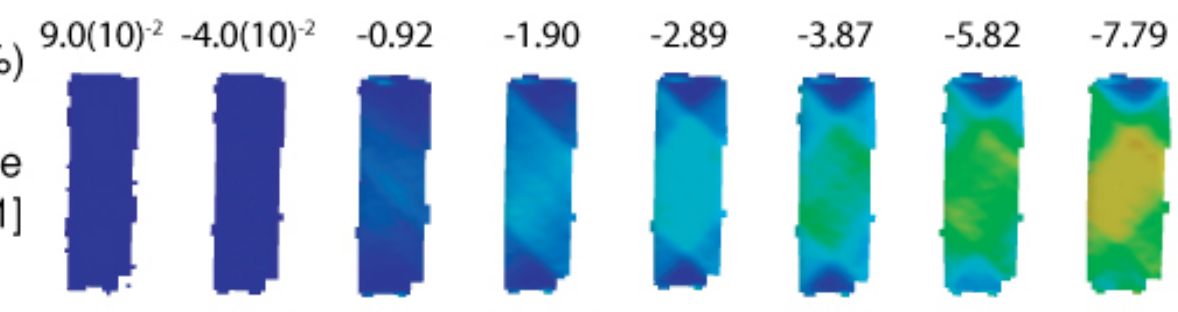

$-10.28$

$y$-Face

[49 22 -5]
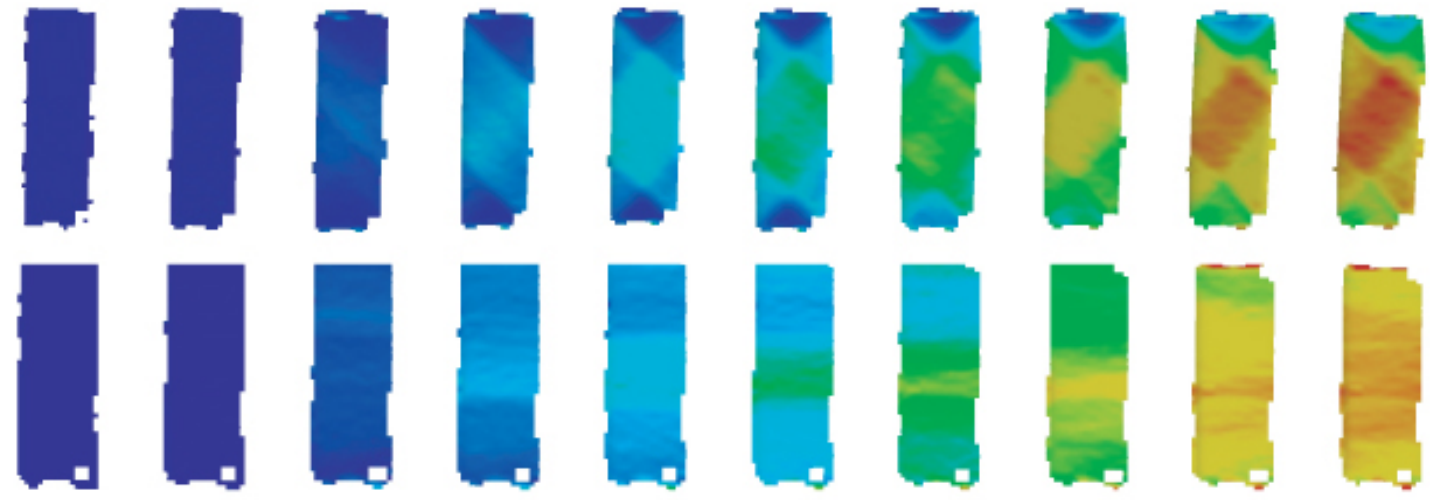

\section{$\%$ Strain

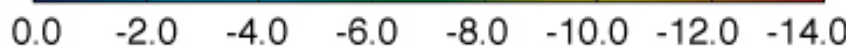

Fig. 4: Stress-strain curve for compressed copper crystal from extensometer data and succession of images showing color-coded image correlation maps of the distribution of compressive strain $\left(\varepsilon_{\mathrm{zz}}\right)$ on the $\mathrm{x}$ - and $\mathrm{y}$-faces as a function of the overall axial strain on the specimen. 

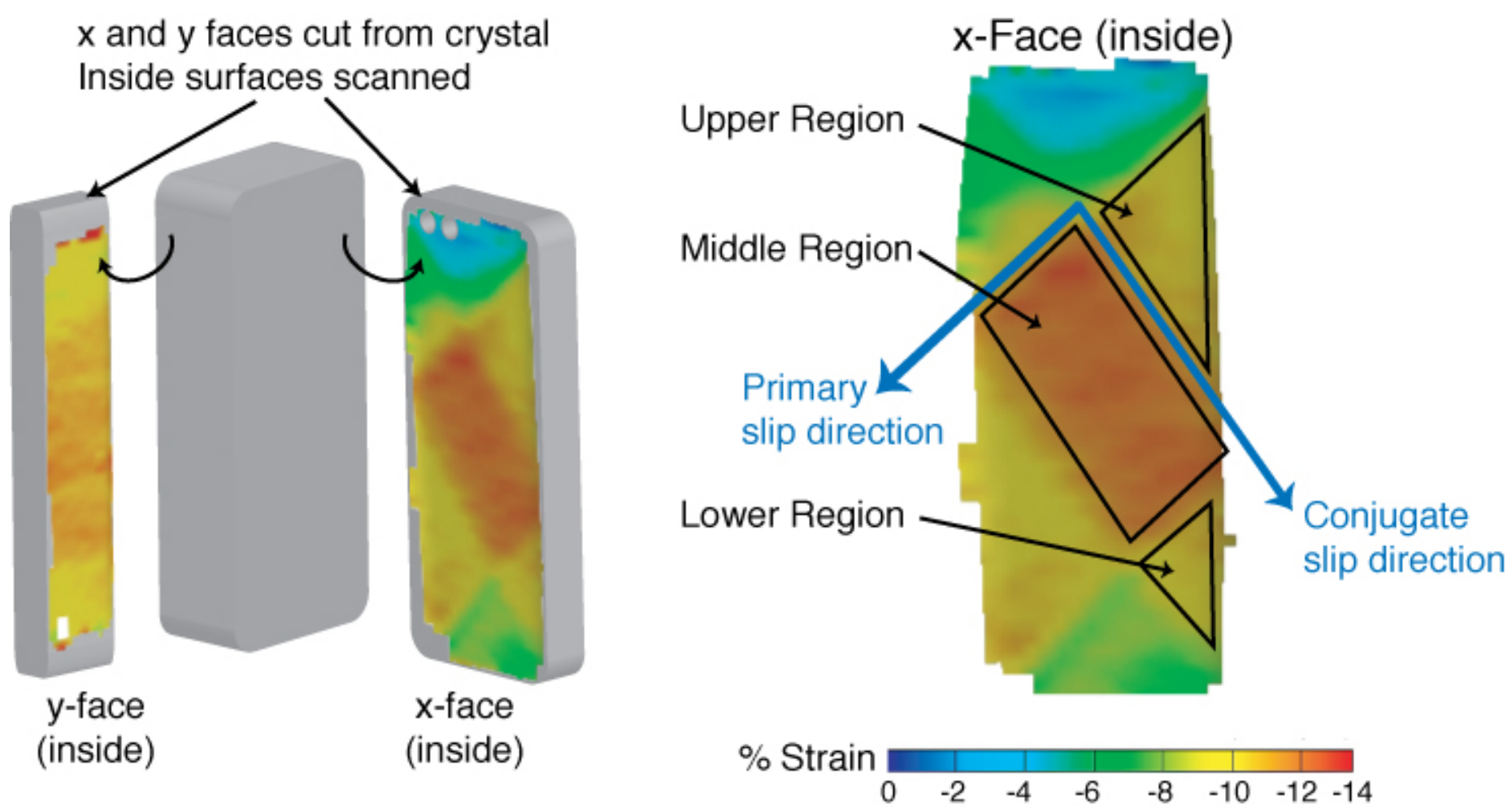

Fig. 5: Model of sample and image correlation strain maps showing the surfaces scanned were the inside surfaces of $1 \mathrm{~mm}$ thick slabs cut from the $\mathrm{x}$ - and $\mathrm{y}$-faces. The distinct regions on the $\mathrm{x}$-face are shown. Note the strain is a mirror image of that shown in Fig. 4. 


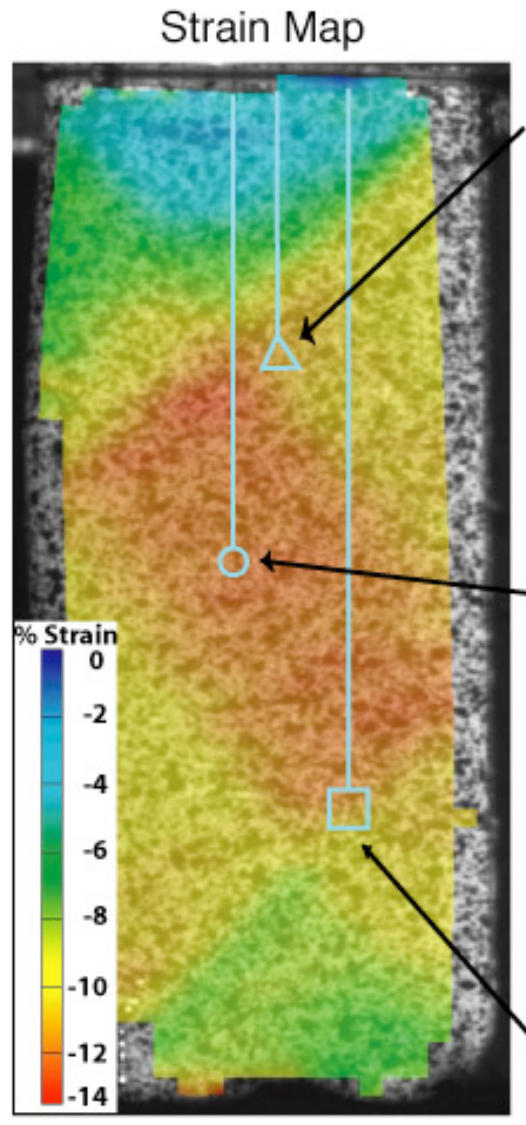

Top Pt placed $3.82 \mathrm{~mm}$ from top of crystal

Pt arrow placed $6.61 \mathrm{~mm}$ from top of crystal

Bottom Pt mark placed $10.17 \mathrm{~mm}$ from top of crystal
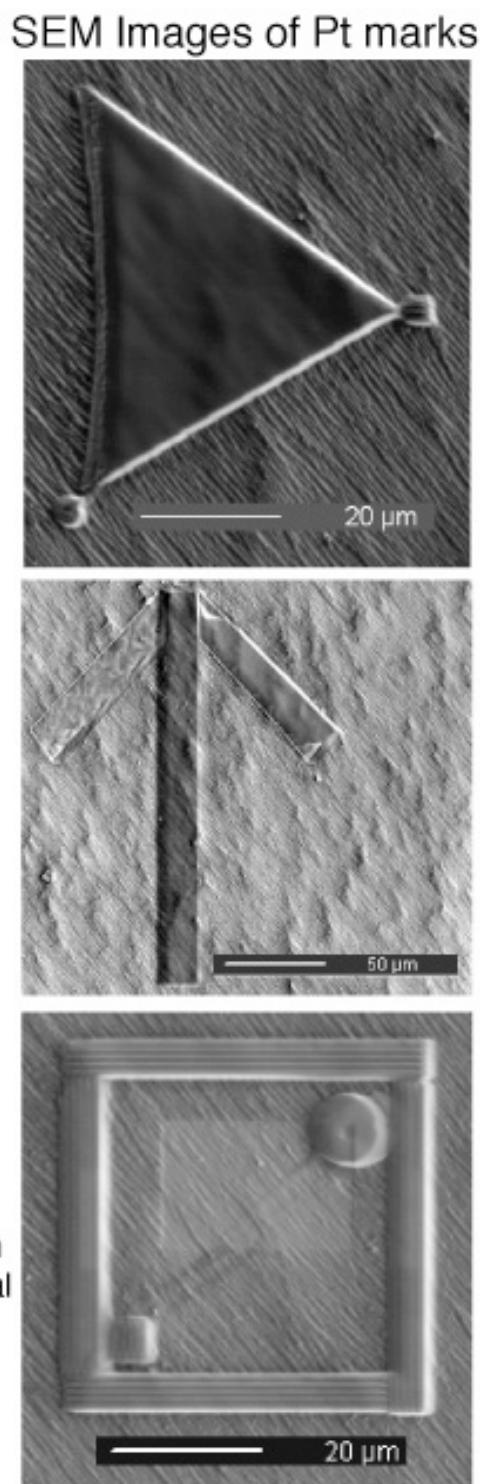

X-ray microfluorescence map

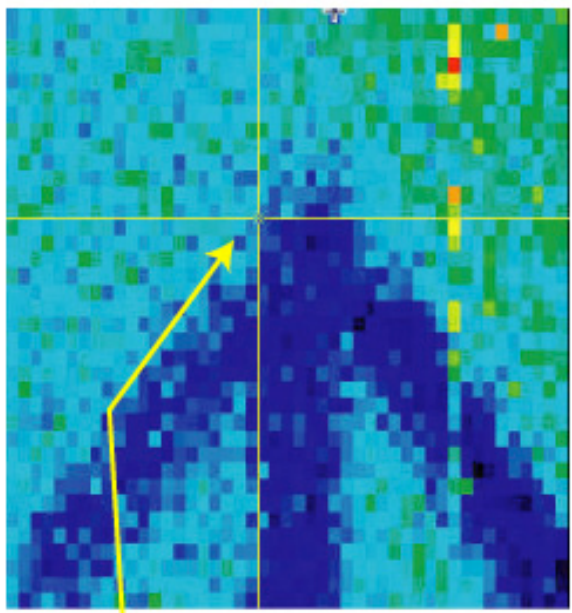

Reference point for microdifraction scans

Fig. 6: The image correlation strain map with locations of platinum markers shown. SEM images of the three platinum markers are shown, along with the x-ray microfluorescence map of the platinum arrow (central marker) taken on the beamline.
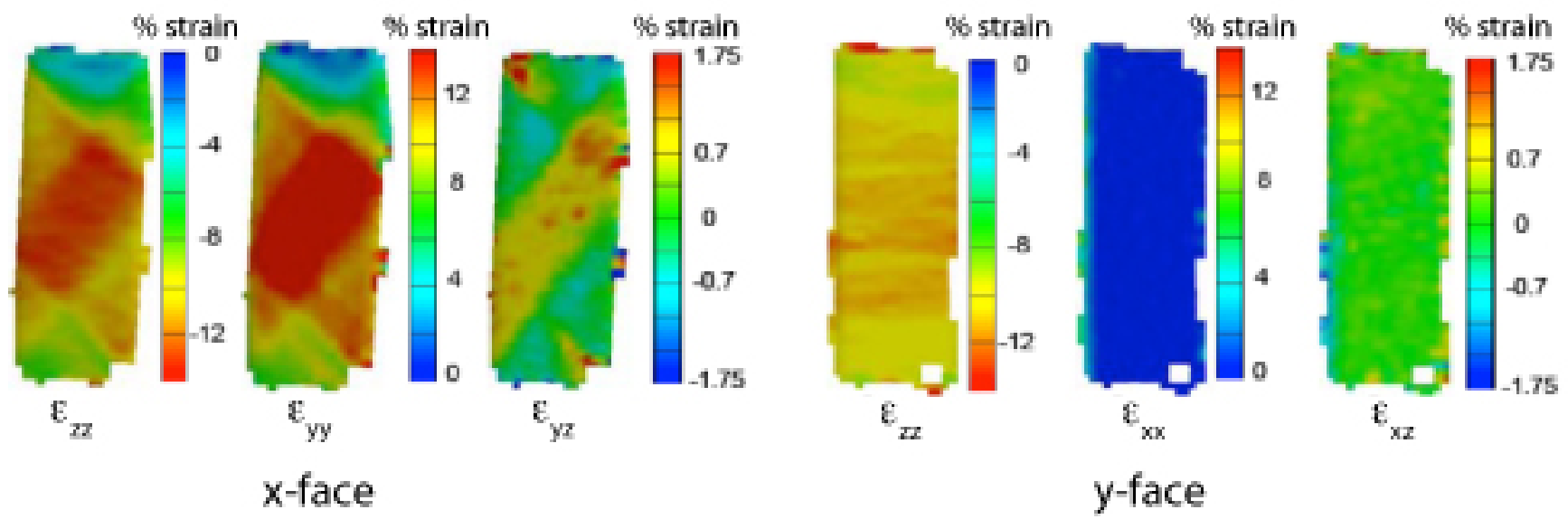

Fig. 7: Image correlation maps of the strain fields in the $x$ - and $y$-faces. 

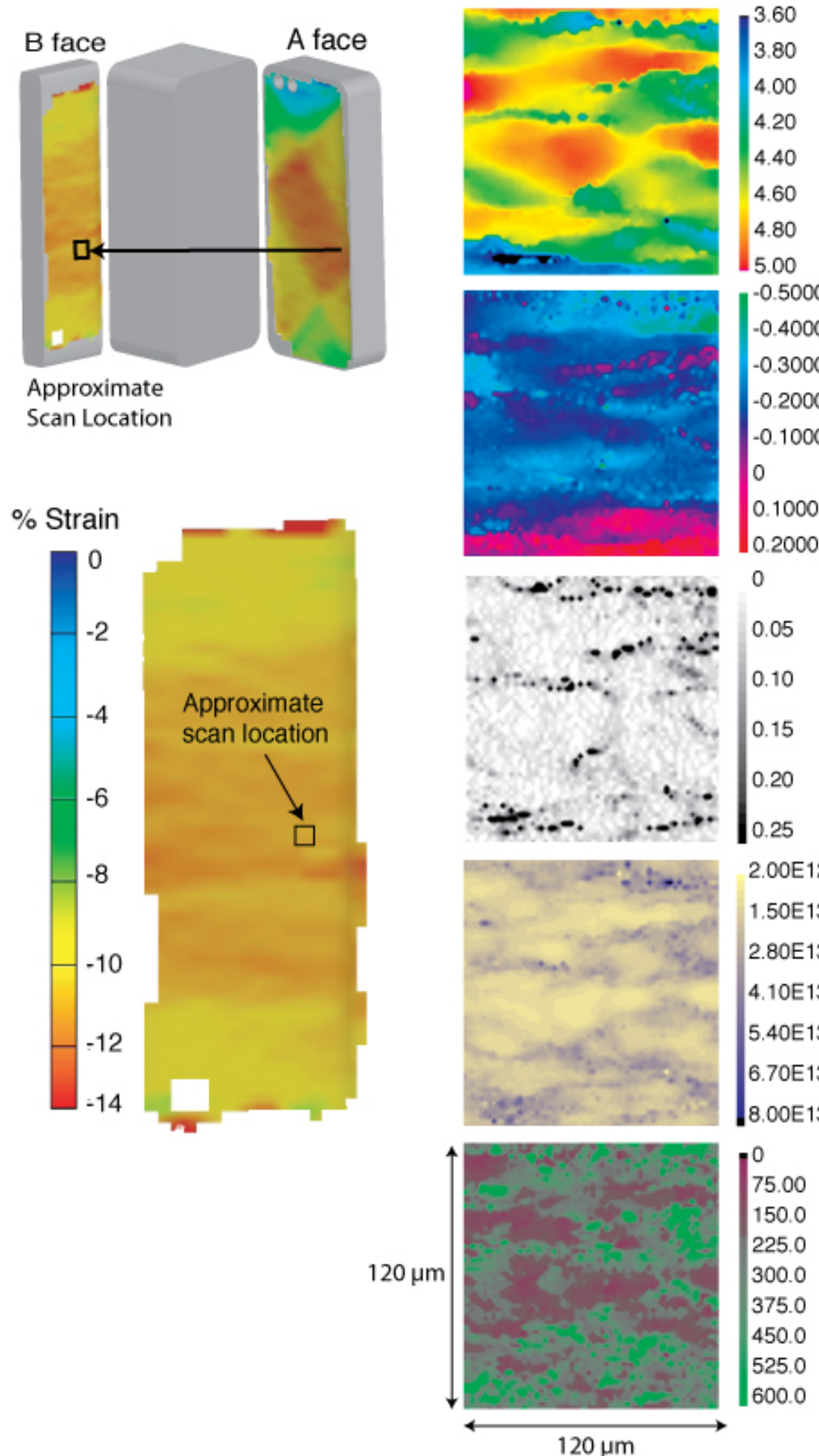

(a) Out-of-Plane Orientation Angle $\left({ }^{\circ}\right)$

4.80

5.00

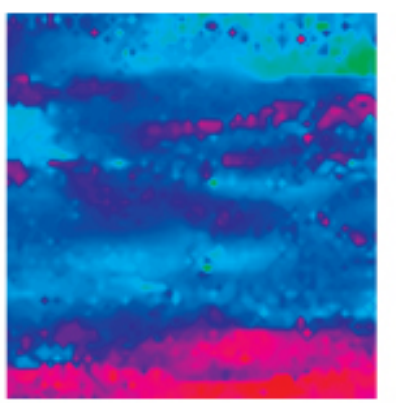

$=0.5000$

$-0.4000$

$-0.3000$

$-0.2000$

$-0.1000$

0

0.1000

0.2000
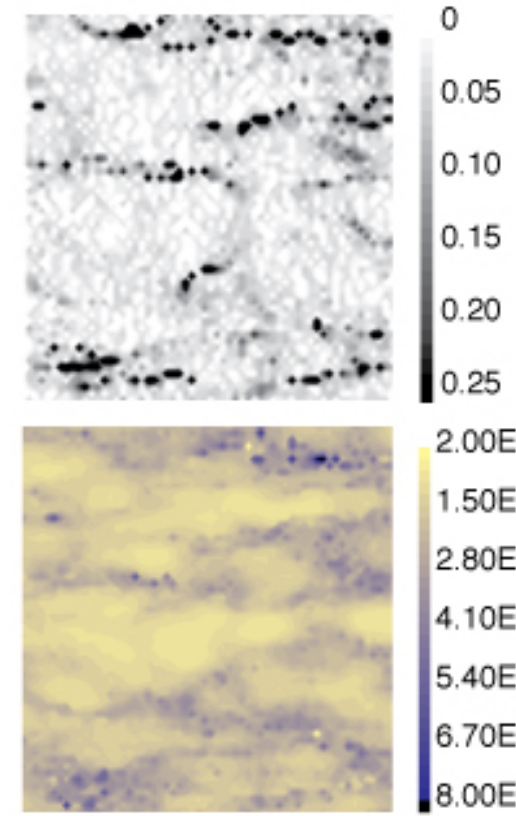

2.00E12

$1.50 \mathrm{E} 13$

2.80E13

4.10E13 (d) Dislocation

$5.40 \mathrm{E} 13$ Density $\left(\mathrm{m}^{-2}\right)$

$6.70 \mathrm{E} 13$

$8.00 E 13$

(c) Misorientation

Angle $\left({ }^{\circ}\right)$

(b) In-Plane

Orientation

Angle $\left({ }^{\circ}\right)$

(e) Maximum

Resolved Shear

Stress (MPa)

Fig. 8: Microdiffraction maps from the y-face, taken with 2-micron step size. The approximate location on strain maps is noted. Note that the bands of high dislocation density superimpose on gradients in the out-of-plane orientation. 


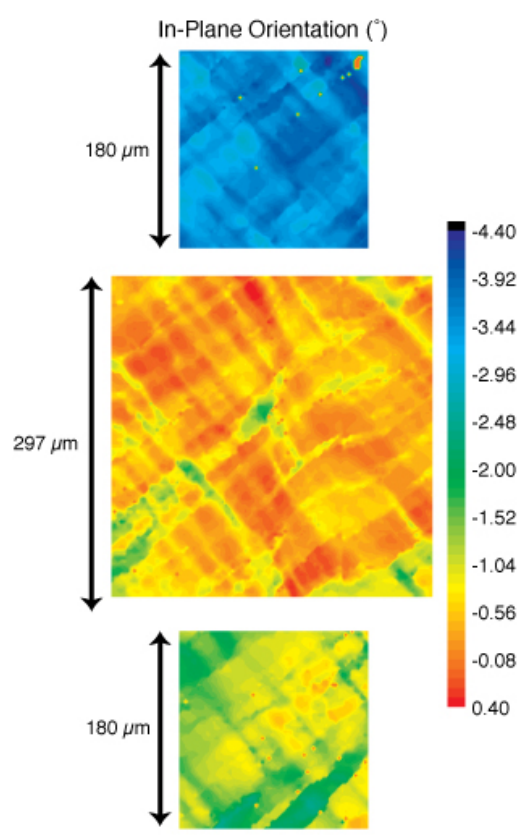

(a)

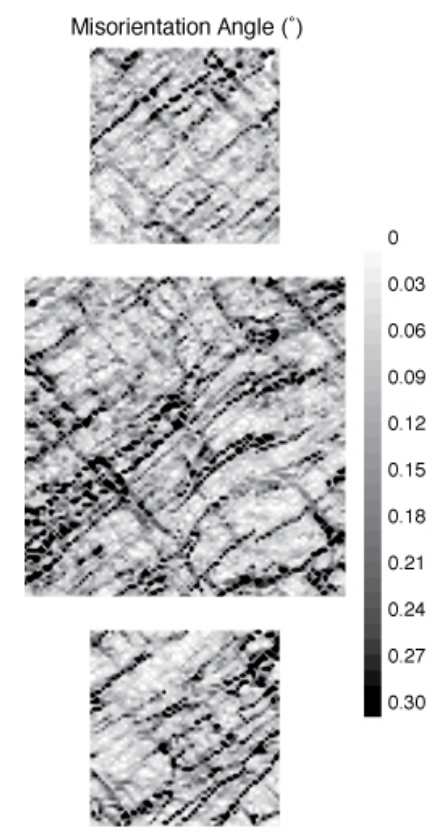

(b)

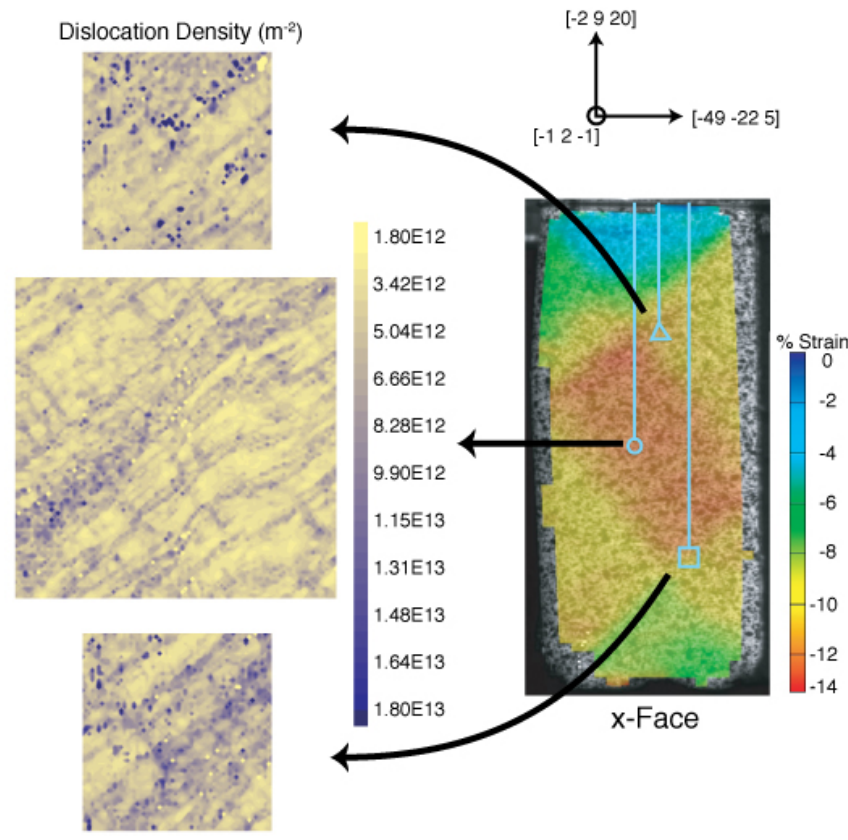

(c)

Fig. 9: Microdiffraction maps from large area scans in 3 regions on the x-face noted in strain map, taken with 3-micron step size: (a) in-plane orientation, (b) in-plane misorientation angle maps and (c) dislocation density maps. Orthogonal sets of parallel bands of high dislocation density and large misorientation are seen throughout the crystal.

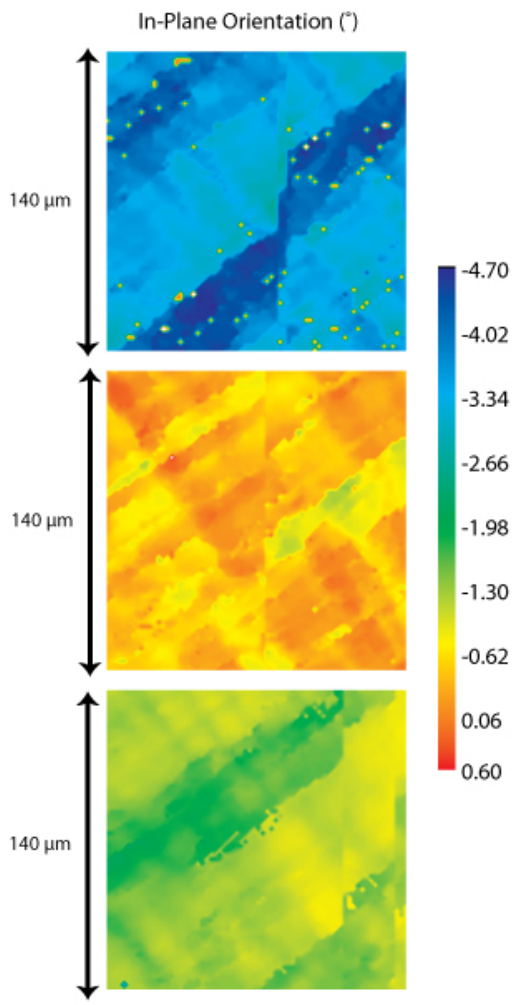

(a)

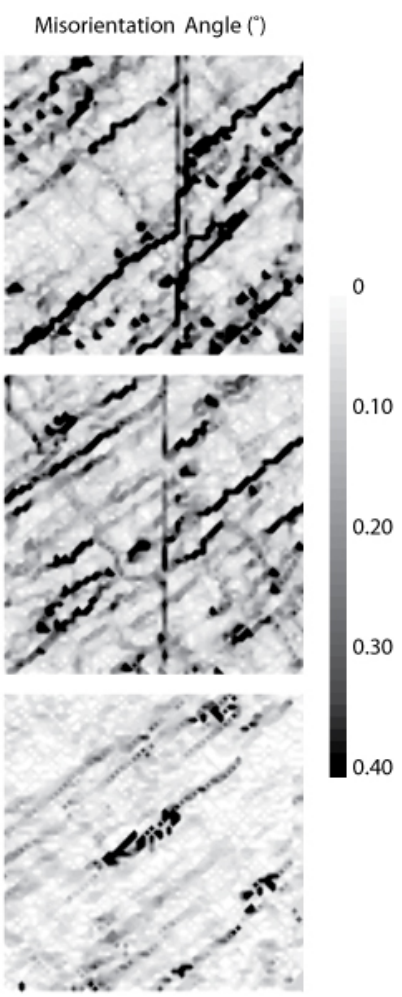

(b)

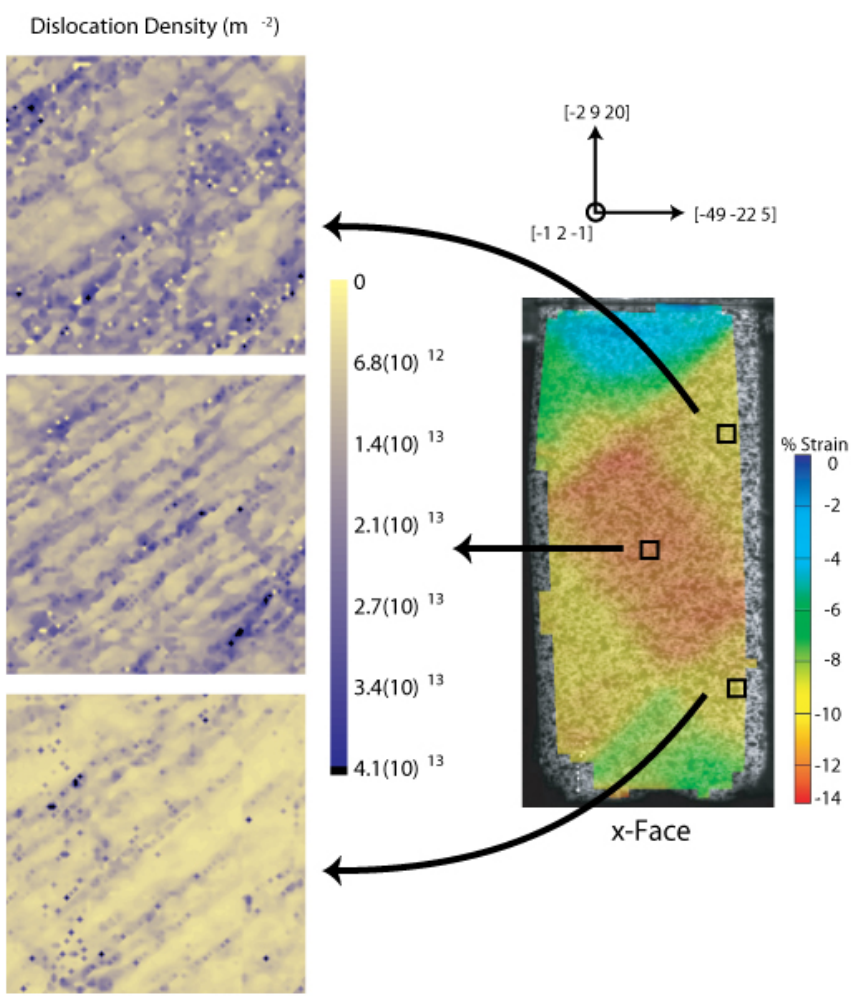

(c)

Fig. 10: Microdiffraction maps from large area scans in 3 regions on the $x$-face noted in strain map. Locations chosen so to present data that is more clearly from within the primary and conjugate bands than in Figure 9. Discontinuities in maps are experimental artifacts. 


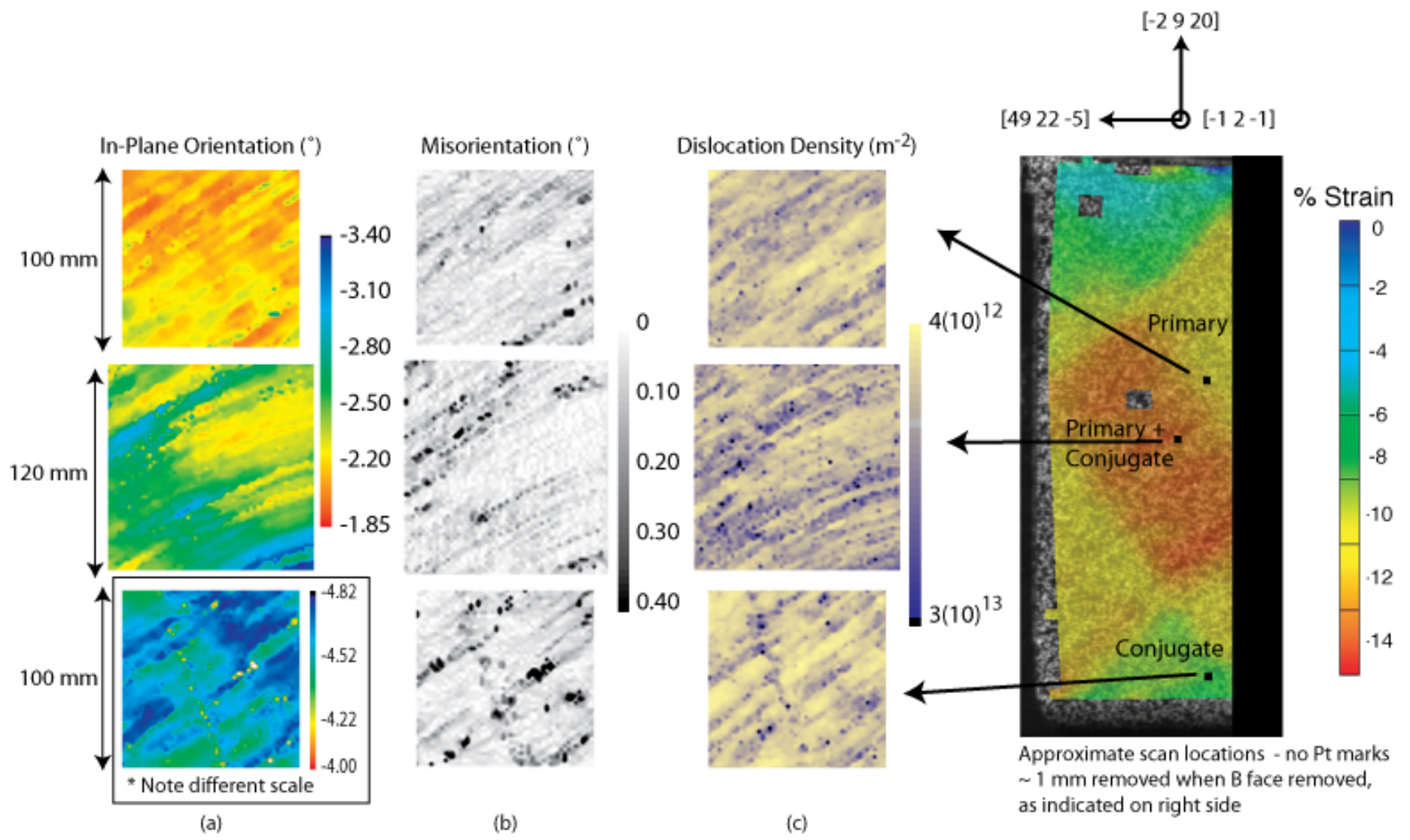

Fig. 11: Microdiffraction maps taken from opposite face of the tested specimen, approximate locations on strain maps are noted. Sample face underwent no mechanical cutting or polishing post-deformation. 


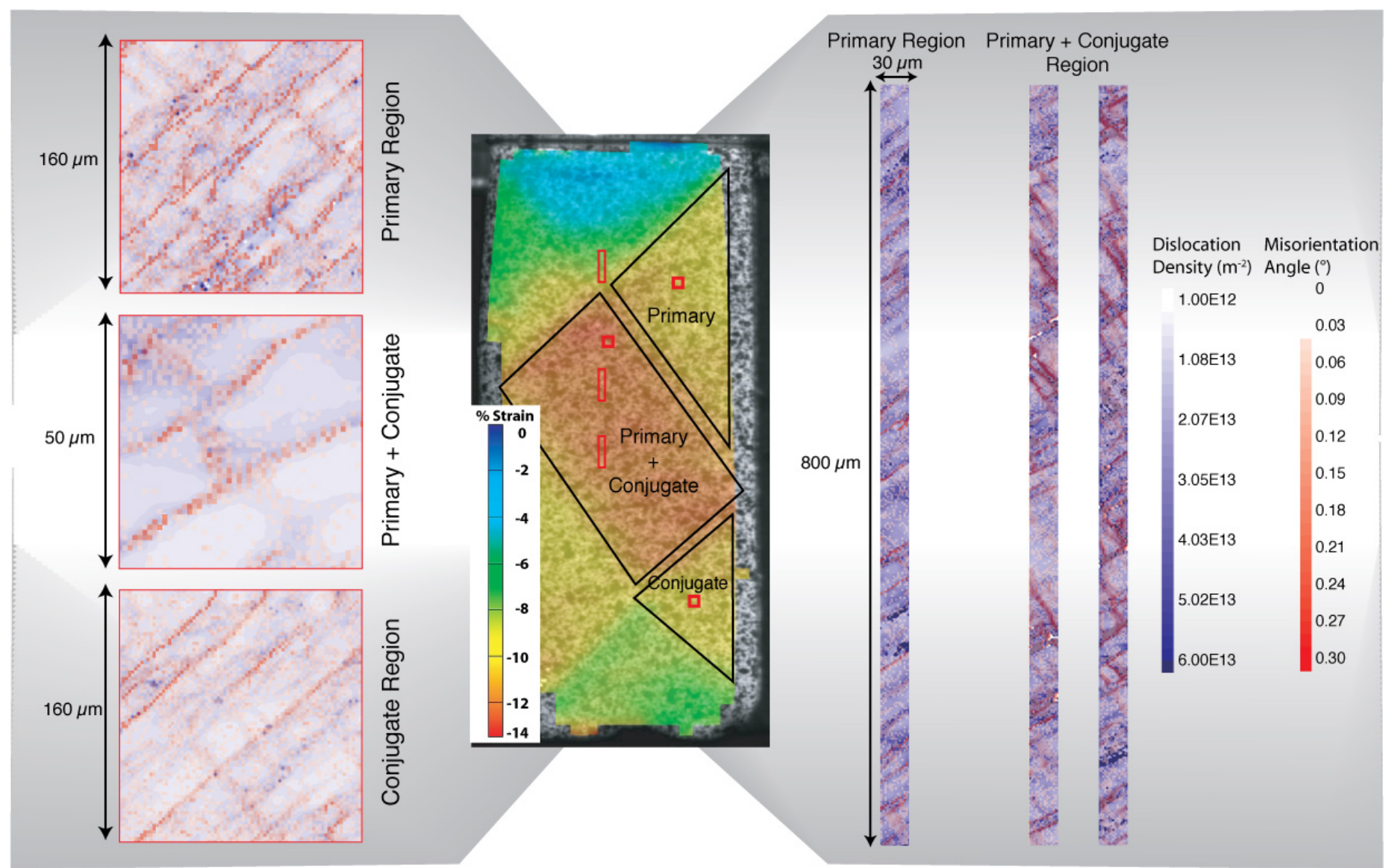

Fig. 12: Maps of misorientation angle superimposed on maps of dislocation density to demonstrate the suitability of the dislocation density approximation. This approximation calculates the dislocation density from the average peak-width assuming the peak spreading is caused by lattice curvature due to excess same-sign dislocations. The square area scans are taken from each of the strained regions of the crystal, and the strips of surface mapped are $800 \times 30 \mu \mathrm{m}^{2}$, one taken from the primary deformation band, two from the region of mixed primary and conjugate deformation. 


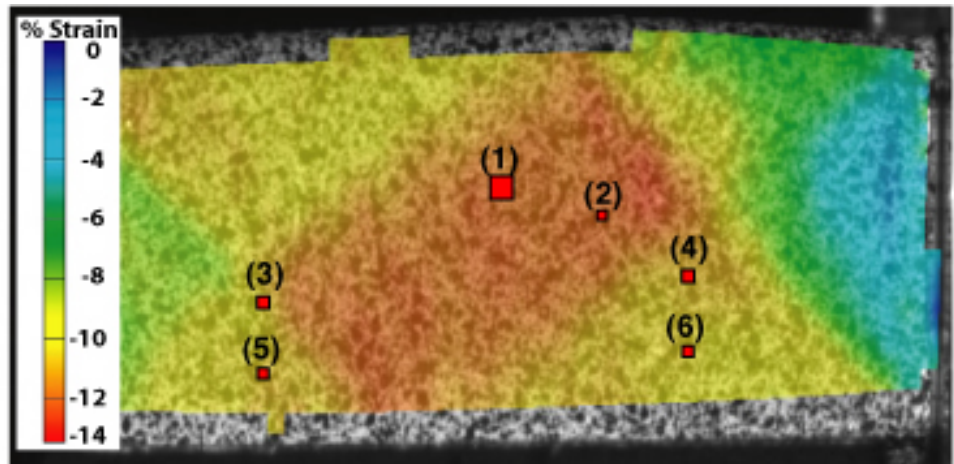

(1) Primary \& Conjugate region $297 \times 297 \mu \mathrm{m}, 3 \mu \mathrm{m}$ step

Dislocation Den
$\left(\mathrm{m}^{2}\right)$
$2.00 \mathrm{E} 12$
$1.20 \mathrm{E} 13$
$2.20 \mathrm{E} 13$
$3.20 \mathrm{E} 13$
$4.20 \mathrm{E} 13$
$5.20 \mathrm{E} 13$
$6.20 \mathrm{E} 13$
$7.20 \mathrm{E} 13$
$8.00 \mathrm{E} 13$

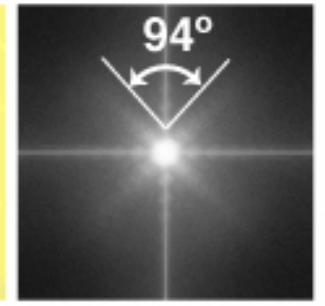

(3) Conjugate region $180 \times 180 \mu \mathrm{m}, 3 \mu \mathrm{m}$ step

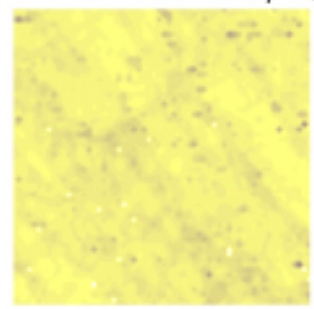

(5) Conjugate region $160 \times 160 \mu \mathrm{m}, 2 \mu \mathrm{m}$ step

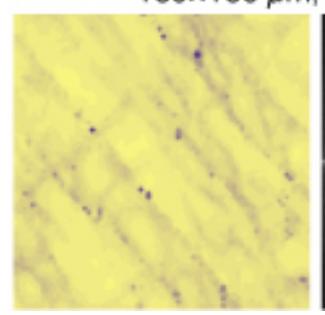

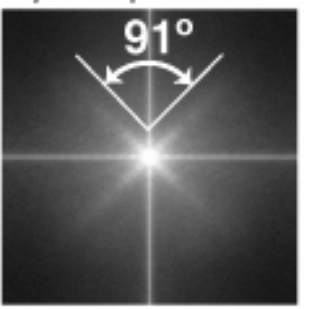

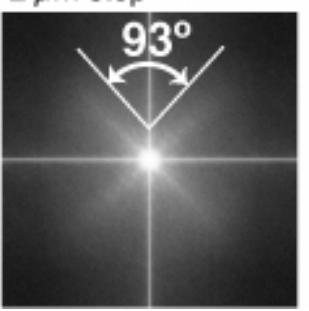

(2) Primary \& Conjugate region $50 \times 50 \mu \mathrm{m}, 1 \mu \mathrm{m}$ step
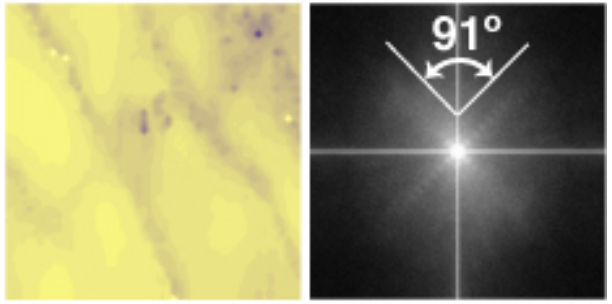

(4) Primary region $180 \times 180 \mu \mathrm{m}, 3 \mu \mathrm{m}$ step

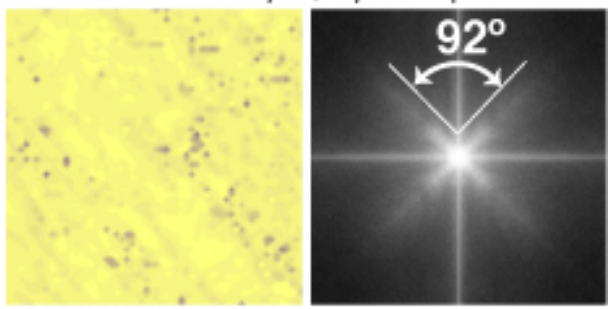

(6) Primary region $160 \times 160 \mu \mathrm{m}, 2 \mu \mathrm{m}$ step

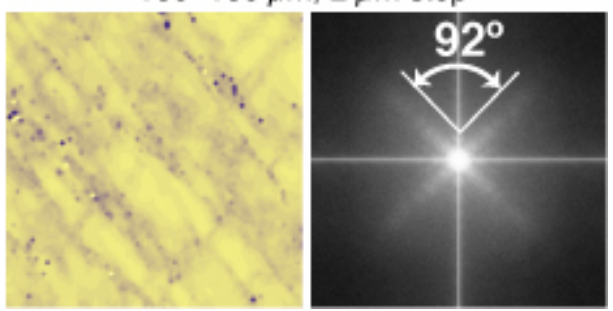

Fig. 13: Maps of dislocation density from x-ray microdiffraction results and corresponding Fourier transforms of each map. The angle of intersection between the primary and conjugate is approximately 90 degrees in all regions of the crystal. 

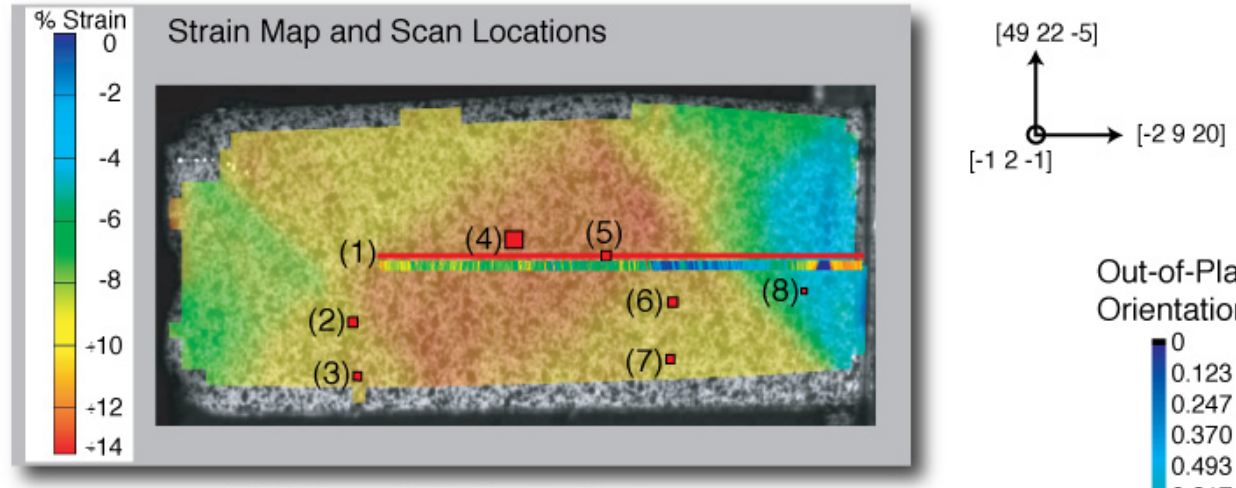

(4) Primary \& Conjugate Region

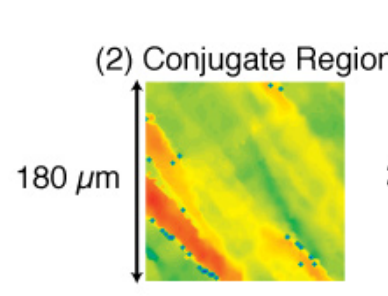

(1) Along long-length scan $\left(0.030 \times 9.592 \mathrm{~mm}^{2}\right)$
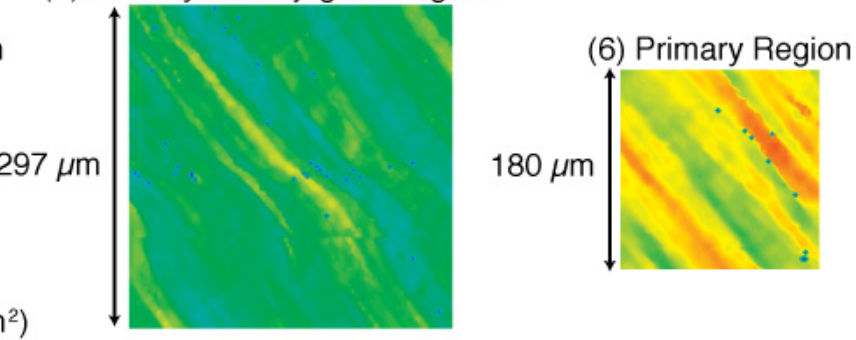

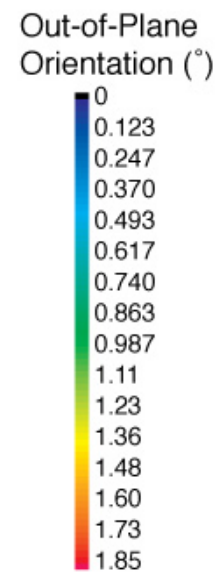

(3) Detail in Conjugate region

(7) Detail in Primary region

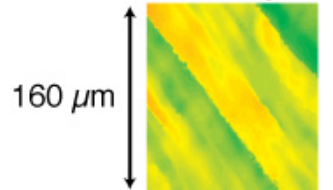

(5) Detail in Middle region

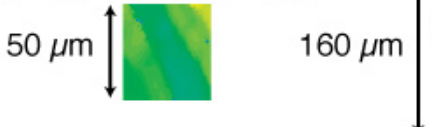

(8) Detail in Top region

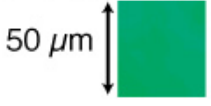

Fig. 14: Out-of-plane orientation maps from area scans and long-length scan linked to locations on strain map. Scans (2), (4) and (6) were taken with a $3 \mu \mathrm{m}$ step-size; scans (1), (3), (7), and the long-length scan with a $2 \mu \mathrm{m}$ step-size; and scans (5) and (8) with a 1 $\mu \mathrm{m}$ step size. 


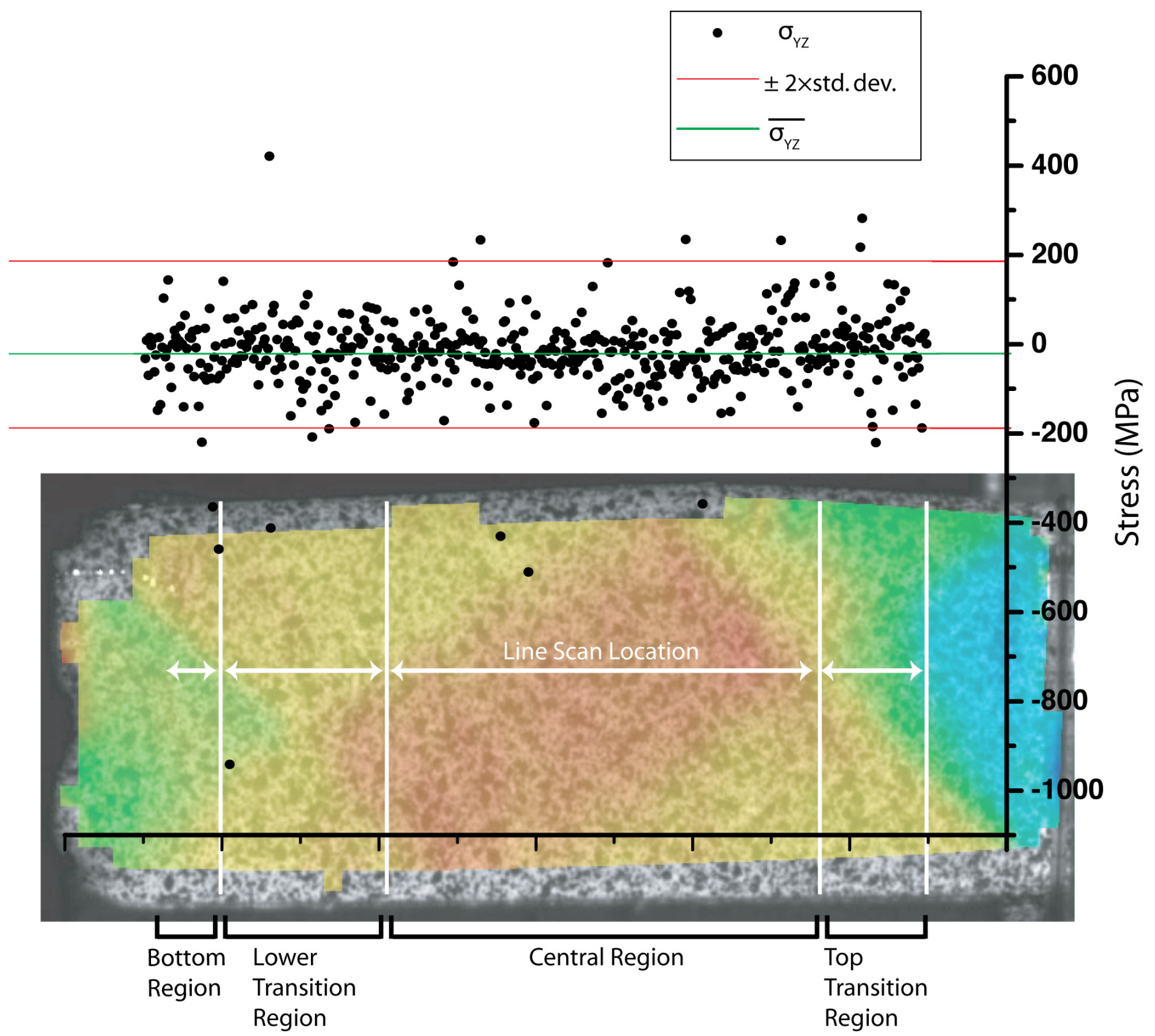

Fig. 15a: (a) Plot of the in-plane shear stress in the $\mathrm{x}$-face $\left(\sigma_{\mathrm{yz}}\right)$ at $20 \mu \mathrm{m}$ intervals along the line drawn in the crystal. The compressive strain map is in the background for reference. The stress scatters over several hundred MPa; the " $2 \sigma$ " limits are drawn. (b) The distribution of shear stress values from the line scan shown. 


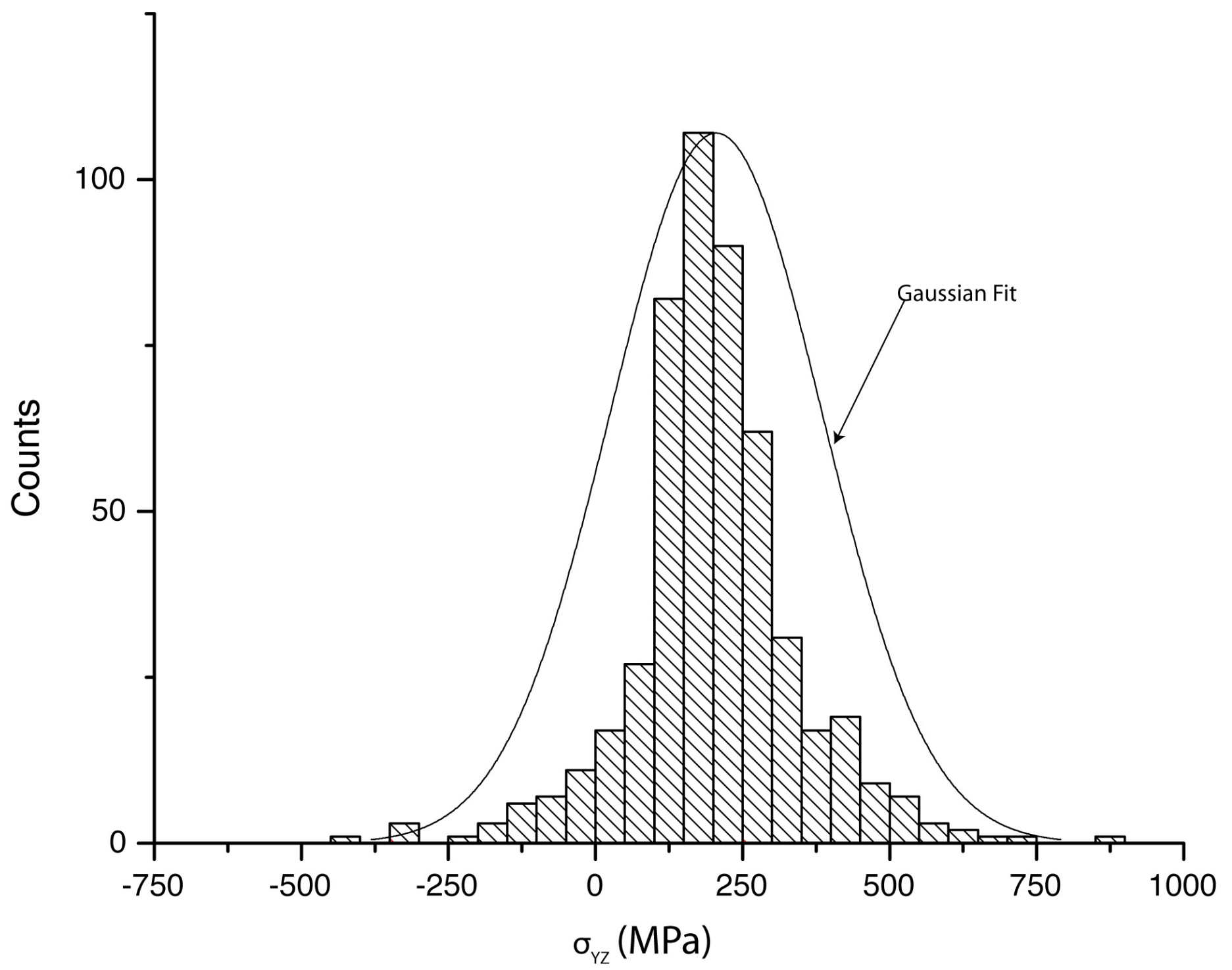

Fig. 15b: The distribution of shear stress values from the line scan shown in (a). 


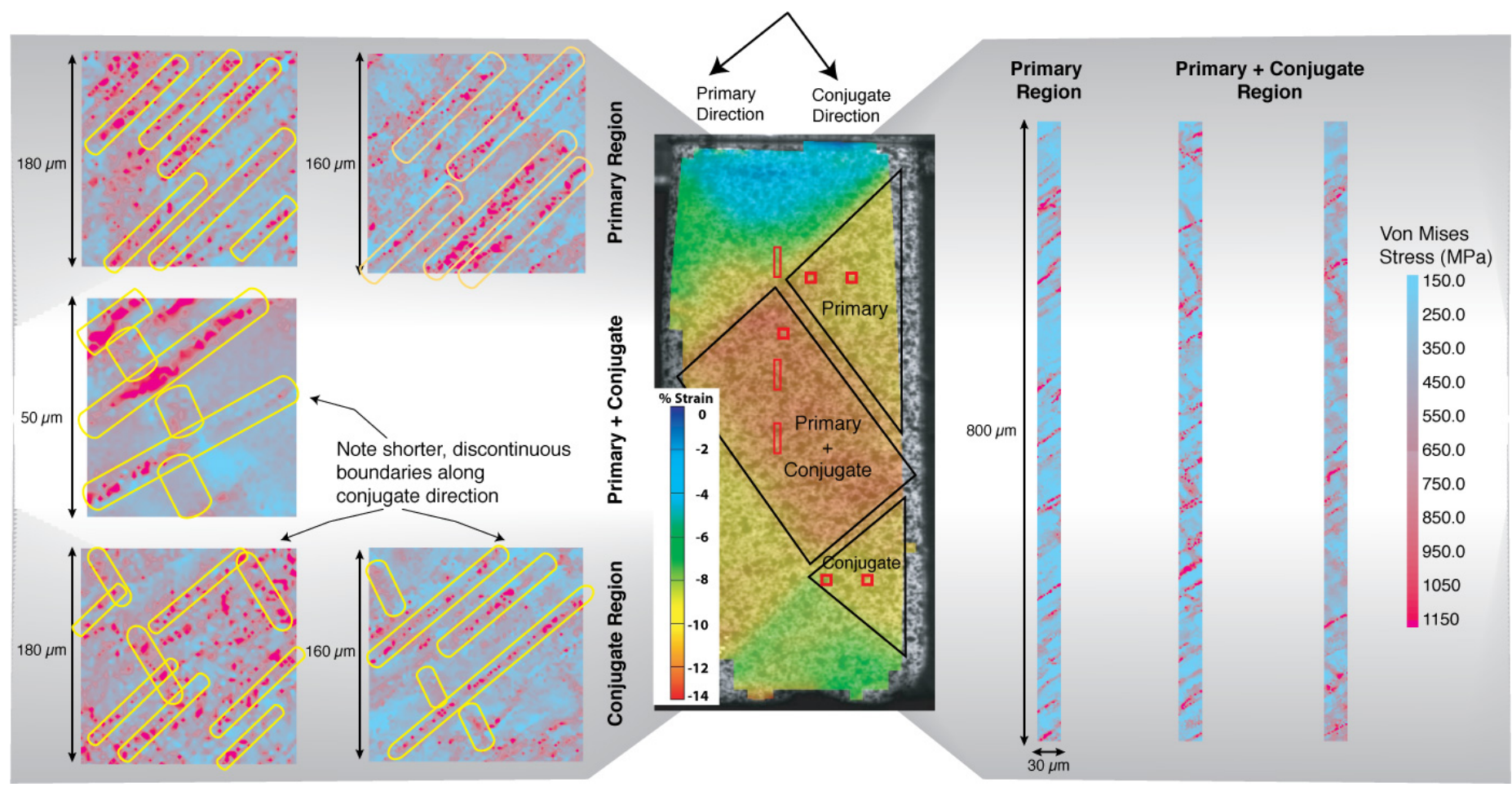

Fig. 16: Maps of the Von Mises shear stress over small areas of the $x$-face taken from the primary, primary + conjugate, and conjugate strain regions, and over $30 \times 800 \mu \mathrm{m}$ strips on the $\mathrm{x}$-face taken from the primary and primary + conjugate regions. Note that the measured residual shear stress reaches $1 \mathrm{GPa}$. 


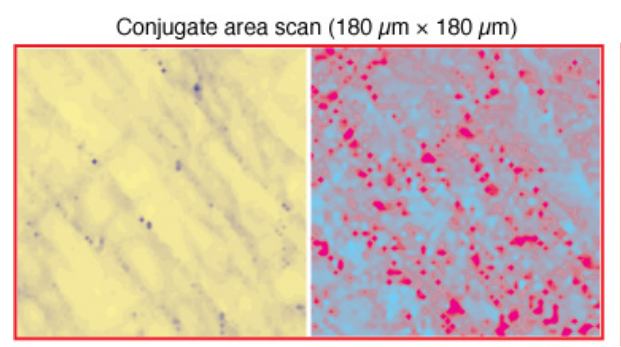

Primary+Conjugate area scan $(50 \mu \mathrm{m} \times 50 \mu \mathrm{m})$

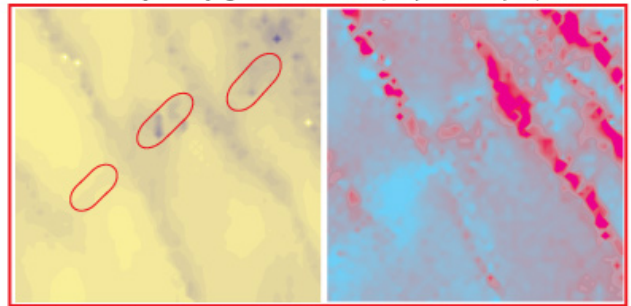

Boundaries along Conjugate Boundaries along Conjugate
direction in offset segments

Dislocation
Density $\left(\mathrm{m}^{-2}\right)$ 2.00E12

$1.20 \mathrm{E} 13$

$2.20 \mathrm{E} 13$

$3.20 \mathrm{E} 13$

$4.20 \mathrm{E} 13$

$5.20 \mathrm{E} 13$

$6.20 \mathrm{E} 13$

$7.20 \mathrm{E} 13$

8.00E13

(1) Boundaries along primary and conjugate directions
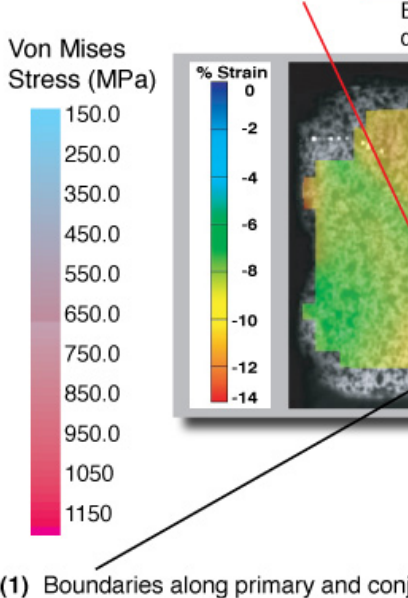
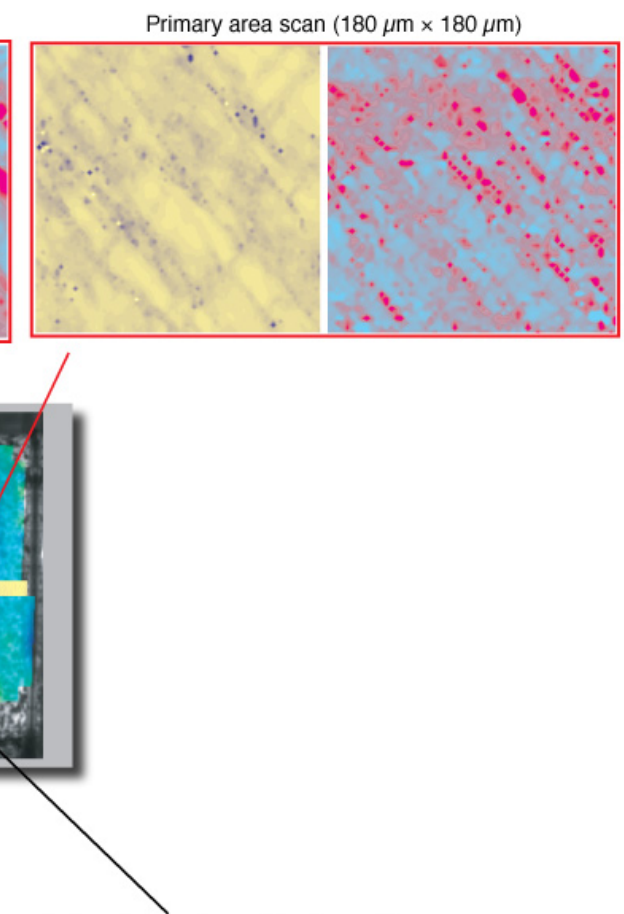

(3) Boundaries along primary direction only

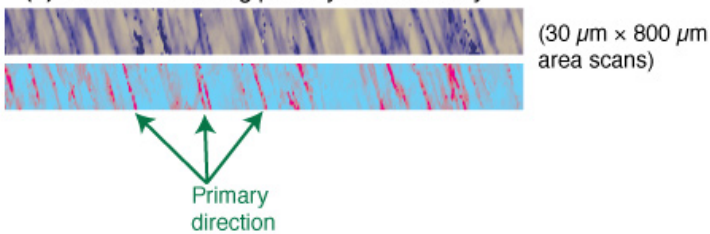

Fig. 17: Comparative maps of the average dislocation density and Von Mises stress for the three regions of strain in the crystal. Bands of high stress and high dislocation density superimpose clearly for the primary shear bands. The superposition is present, but less obvious for the conjugate shear bands. 


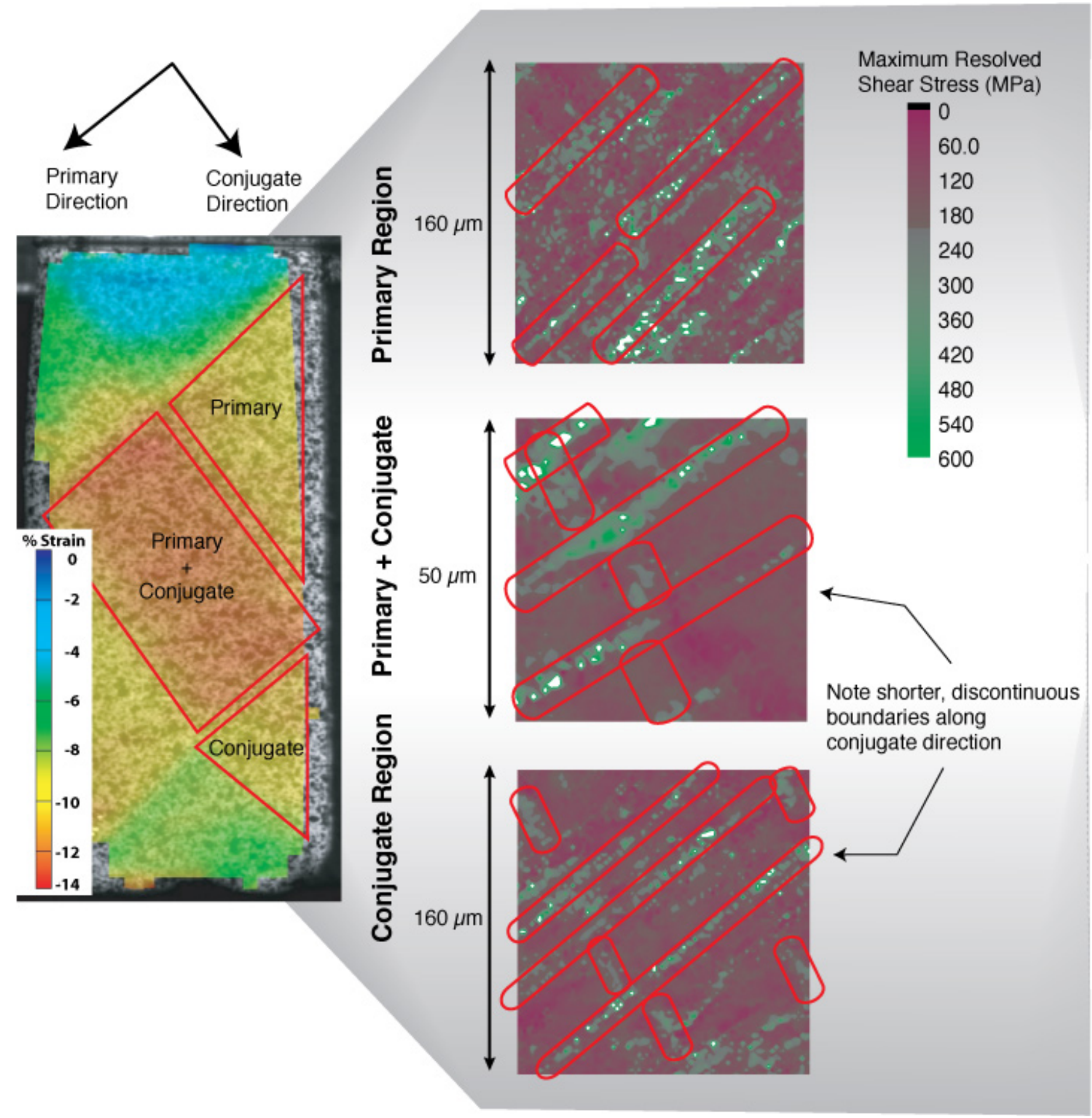

Fig. 18: Maps of the maximum resolved shear stress over regions taken from the primary, primary + conjugate and conjugate shear regions. Note that the bands of maximum stress that parallel bands of conjugate shear are relatively diffuse and discontinuous. 


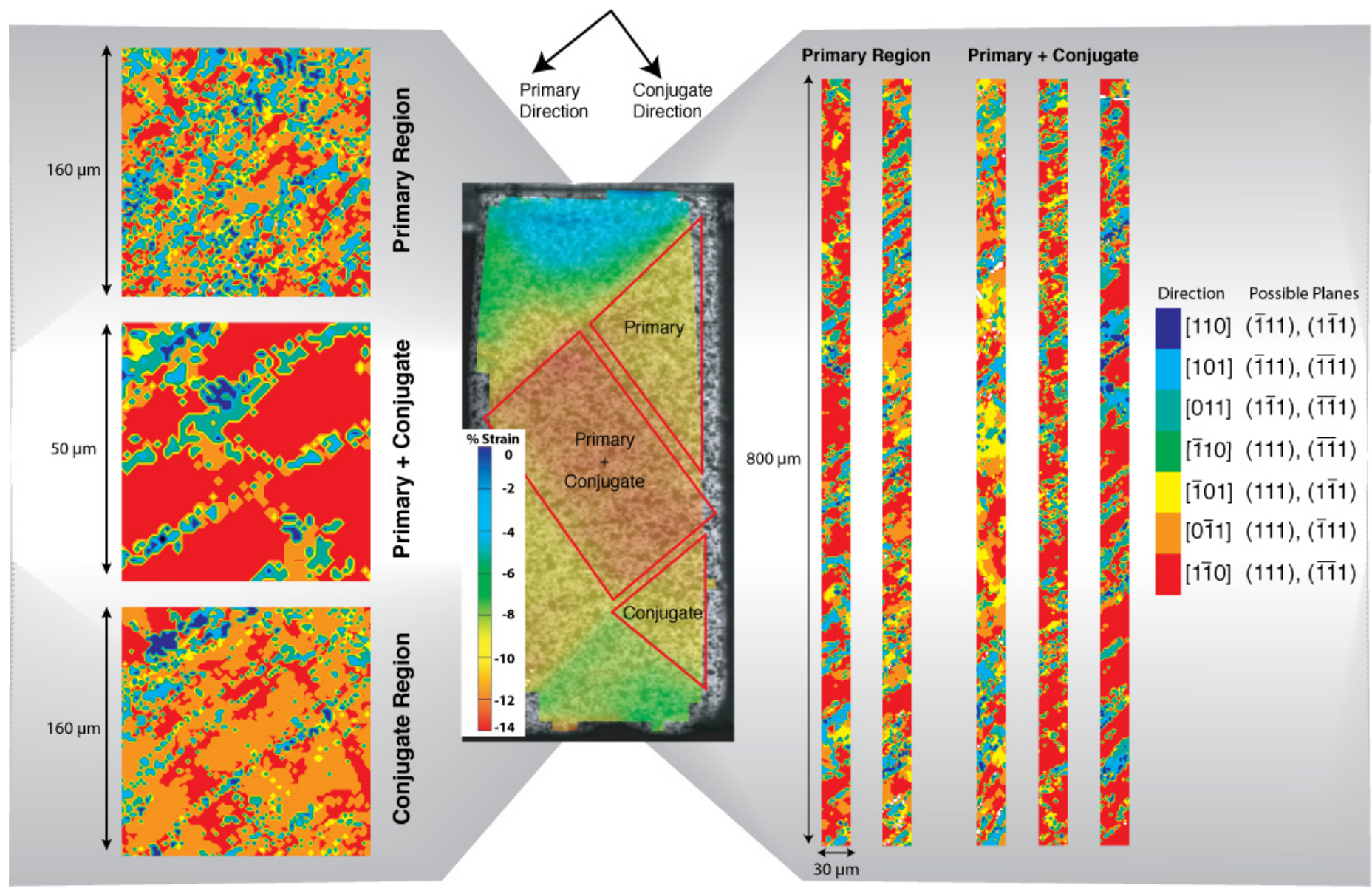

Fig. 19: Maps of the crystallographic direction of the maximum resolved shear, along with the possible planes that may contain this direction. Note that the highest stresses lie in bands parallel to the primary (111) slip planes, but in directions that are not contained in these planes. 


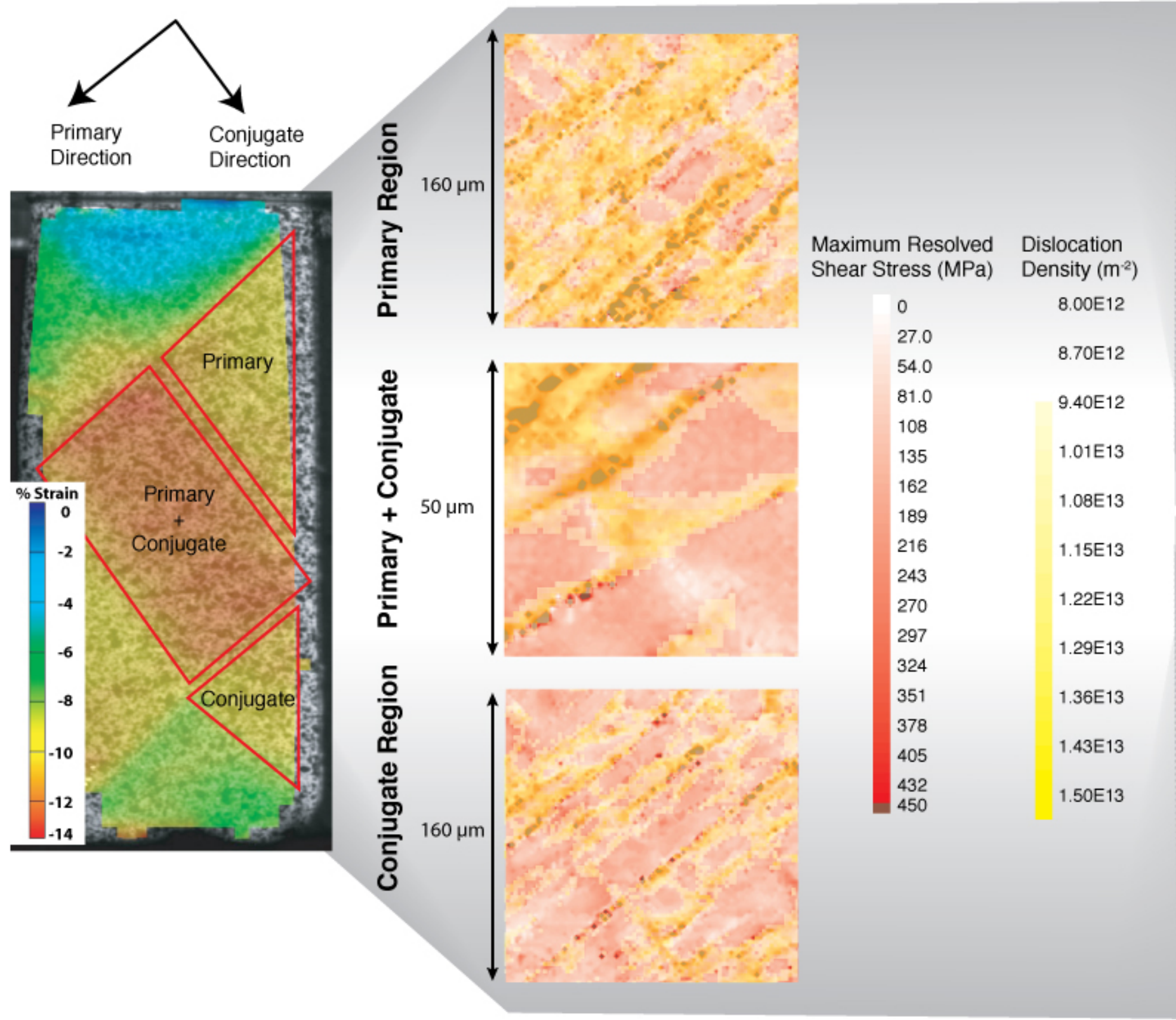

Fig. 20: Dislocation density maps overlaid on MRSS maps, demonstrating correspondence between high dislocation density and maximum shear stresses. This correspondence indicates that the dislocations are imposing the shear stresses rather than relaxing them. 